Article

\title{
Phytochemical Screening and Acanthamoebic Activity of Shoots from in Vitro Cultures and in Vivo Plants of Eryngium alpinum L.-The Endangered and Protected Species
}

\author{
Małgorzata Kikowska ${ }^{1, *(\mathbb{D})}$, Dariusz Kruszka ${ }^{2}\left(\mathbb{0}\right.$, Monika Derda $^{3}$, Edward Hadaś ${ }^{3}(\mathbb{D}$ and \\ Barbara Thiem ${ }^{1}$ \\ 1 Department of Pharmaceutical Botany and Plant Biotechnology, Poznan University of Medical Sciences, 14 \\ Św. Marii Magdaleny St., 61-861 Poznań, Poland; bthiem@ump.edu.pl \\ 2 Institute of Plant Genetics, Polish Academy of Sciences, 34 Strzeszyńska St., 60-479 Poznań, Poland; \\ dkru@igr.poznan.pl \\ 3 Department of Biology and Medical Parasitology, Poznan University of Medical Sciences, 10 Fredry St., \\ 61-701 Poznań, Poland; mderda@ump.edu.pl (M.D.); ehadas@ump.edu.pl (E.H.) \\ * Correspondence: kikowska@ump.edu.pl; Tel.: +48-61-668-78-50
}

Received: 24 February 2020; Accepted: 17 March 2020; Published: 20 March 2020

check for updates

\begin{abstract}
Genetically uniform shoots of Eryngium alpinum L. cultured in vitro were subjected to the qualitative analysis applying the UPLC-HESI-HRMS technique. In vitro cultures give the opportunity to perform the phytochemical studies on the protected species without harvesting the plant material from the natural environment. The phytochemical screening of the crude methanolic extracts of shoots, both from in vitro cultures and in vivo plants, revealed the presence of phenolic acids, coumarins, flavonoids, triterpenoid saponins, amino acids, or dipeptides. Active compounds detected are known to have medicinal importance, and for this reason, the present study represents a preliminary investigation of the extracts against pathogenic and opportunistic amoeba. Among the extracts tested, the extract of shoots from in vitro cultures exhibited remarkable amoebicidal action against trophozoites. On the second day of treatment, the extract at the concentrations of $5 \mathrm{mg} / \mathrm{mL}, 2.5$ $\mathrm{mg} / \mathrm{mL}$, and $0.5 \mathrm{mg} / \mathrm{mL}$ showed the highest antiamoebicidal effect: the inhibition of trophozoites reached $81.14 \%, 66.38 \%$, and $54.99 \%$, respectively. To our best knowledge, the present report is the first to show the phytochemical screening and to discuss the antiamoebic activity of Eryngium alpinum L. shoots, both from in vitro cultures and in vivo plants.
\end{abstract}

Keywords: alpine eryngo; in vitro shoot culture; phenolic compounds; triterpenoid saponins; phytochemical analysis; Acanthamoeba treatment

\section{Introduction}

Eryngium alpinum L. is a perennial herb in the Saniculoideae subfamily of the Apiaceae family [1]. It is native the European Alps. The population of the plant is in decline. The species is protected by law: the Habitats Directive; the Convention on the Conservation of European Wildlife and Natural Habitats, the European Habitat Directive of Natura 2000, and the national red lists/books of protected species [2].

Due to the unavailability of the plant material, little research on this taxon was carried out. Only a few papers indicated the presence of phenolic acids, flavonoids and the essential oil in the organs of in vivo plants [3-7]. The identification of flavonoids, namely quercetin and kaempferol, in leaves of alpine eryngo was described by Crowden et al. [3]. Moreover, isoquercetin and quercitrin were detected 
in shoots of in vivo plants and in vitro shoot cultures [6,7]. Roots, phytochemically investigated in the study of Le Claire et al., are known to contain chlorogenic acid, R-(+)-rosmarinic acid and its derivative - R-(+)-3'-O- $\beta$-D-glucopyranosyl rosmarinic acid [4]. Caftaric acid, chlorogenic acid, neochlorogenic acid, isochlorogenic acid, 3,4-dihydroxyphenylacetic acid, caffeic acid, and rosmarinic acid were detected in the leaves of in vivo plant and in vitro shoot cultures [6,7]. The dominant components identified in the essential oil of the aerial part of the plant were caryophyllene, bicyclogermacrene, germacrene, cariophyllene oxide, $\alpha$-bisabolol, and camphor. Furthermore, the essential oil showed a promising antiphytoviral effect, which is probably correlated with a high content of $\beta$-caryophyllene and caryophyllene oxide [5].

In vitro cultures of E. alpinum are a part of the collection of the protected species of the Department of Pharmaceutical Botany and Plant Biotechnology of Poznan University of Medical Sciences (PUMS). The use of in vitro cultures of alpine eryngo allows for conducting the phytochemical analyses and testing further biological activities of this species without depleting its natural sites to obtain the plant material. Since the seeds exhibit strong dormancy and a low germination rate, the generative propagation seems to be unsatisfactory to provide the raw material. In this regard, in vitro cultures of a number of the medicinal plant species offer an alternative source of uniform and renewable biomass, usually with high biosynthesis capacity for the desired compounds, with the same optical stereometry as in nature, and thus provide the valuable raw material. An important advantage of in vitro cultures is the possibility of intensifying biosynthesis and affecting the accumulation of desired metabolites in biomass, applying various biotechnological methods. It is possible to obtain biomass in a continuous large-scale production process [8].

Acanthamoeba is a single-celled eukaryote existing in two forms: dormant cysts and vegetative trophozoites. The infective free-living amoeba gains entry into body through eyes and ulcerated skin, which may lead to epithelial and ocular keratitis and granulomatous amoebic encephalitis. Treatment of acanthamoebosis is difficult and not always effective [9-11]. In humans, due to the problems in the treatment of opportunistic and pathogenic Acanthamoeba spp. and the lack of effective but safe drugs, the search continues for substances of plant origin that, applied as combined therapy, could contribute to decreasing the effective doses of antibiotics used [10,12,13].

The aim of the study was to obtain shoot biomass of E. alpinum under in vitro conditions and to conduct phytochemical analysis of the crude extracts as well as to evaluate the activity against Acanthamoeba sp. Shoots were developed from meristematic tissue of lateral buds. Then, they were multiplied in vitro on the artificial media by means of the axillary bud proliferation technique. The protocol of shoot multiplication allows for harvesting the high-quality and uniform raw material from alpine eryngo without decreasing the medicinal quality and quantity of bioactive compounds.

The novelty of this manuscript is the indication of the presence of coumarins, triterpenoid saponins, amino acids, dipeptides, and other compounds for the first time for this species. The manuscript aims to enrich the knowledge of phenolic compounds with unexplored phenolic acids, flavonoids, and their derivatives. The present report is the first to show complete phytochemical screening of this important taxon and discusses the antiamoebic activity of Eryngium alpinum L. shoots, both from in vitro cultures and in vivo plants.

\section{Results and Discussion}

\subsection{In Vitro Shoot Culture}

Eryngium alpinum L. (Figure 1) was introduced into in vitro cultures and shoot cultures were established in our laboratory to study their capability of producing bioactive compounds under controlled conditions [6,7]. The biotechnological parameters of E. alpinum shoot multiplication under controlled conditions are presented in Table 1. 


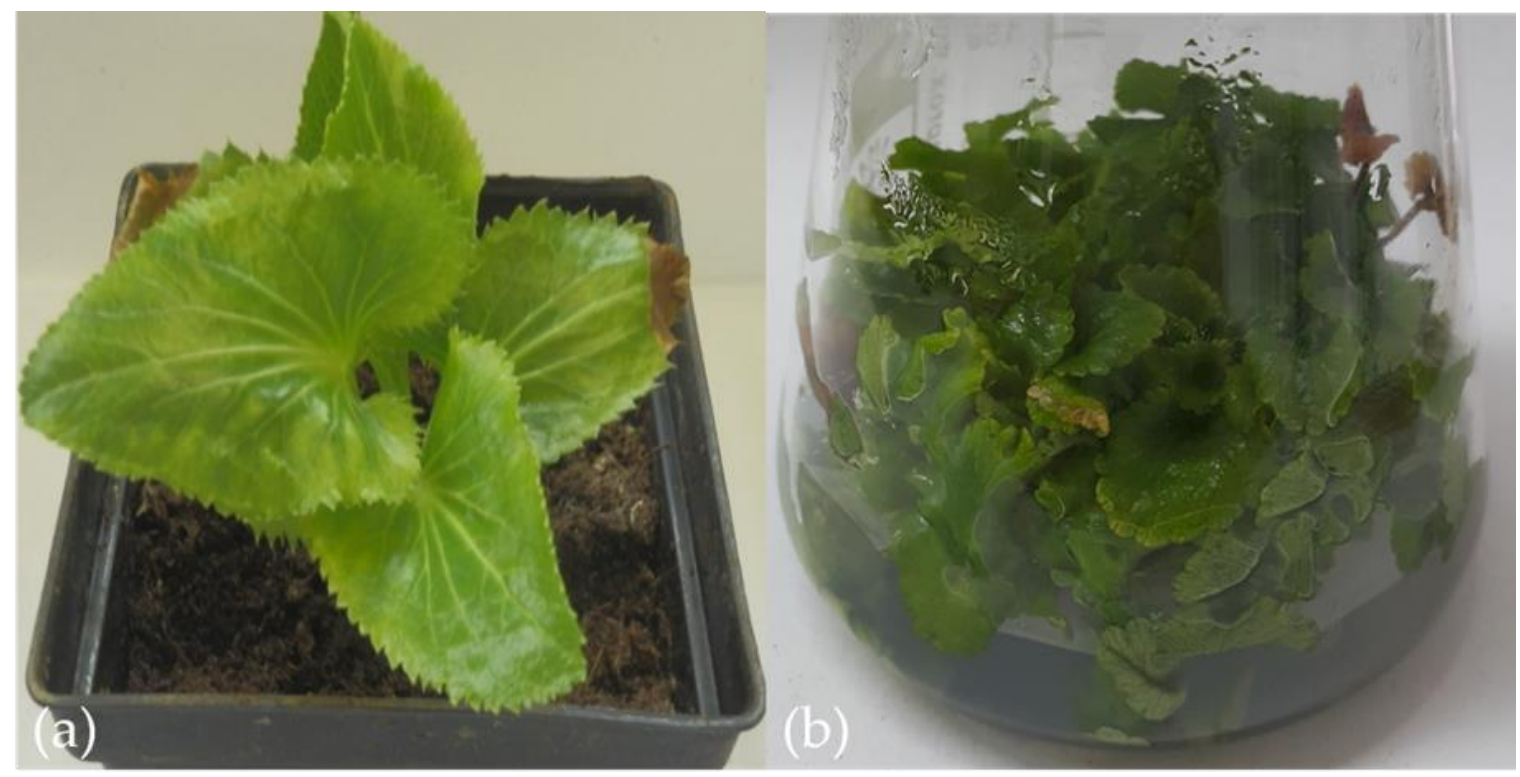

Figure 1. Eryngium alpinum L.: (a) shoots of in vivo plants (b) shoots from in vitro cultures.

Table 1. The effect of selected plant growth regulators-BAP (6-benzylaminopurine), IAA (indole-3-acetic acid) and $\mathrm{GA}_{3}$ (gibberellic acid) present in MS media on shoot multiplication ratio and length of multiplied shoots of Eryngium alpinum L. after 40 days of culture.

\begin{tabular}{cccccc}
\hline No. & $\begin{array}{c}\text { Cytokinin } \\
{[\mathbf{m g} / \mathbf{L}]}\end{array}$ & $\begin{array}{c}\text { Gibberellin } \\
{[\mathbf{m g} / \mathbf{L}]}\end{array}$ & $\begin{array}{c}\text { Auxin } \\
{[\mathbf{m g} / \mathbf{L}]}\end{array}$ & $\begin{array}{c}\text { Mean No. of } \\
\text { Shoots } \pm \text { SE }\end{array}$ & Mean Length of Shoots [cm] \pm SE \\
\hline 1. & BAP 2.0 & $\mathrm{GA}_{3} 1.0$ & - & $6.56 \pm 0.35^{\mathrm{ns}}$ & $2.85 \pm 0.06^{\mathrm{a}, \mathrm{b}}$ \\
2. & BAP 2.0 & $\mathrm{GA}_{3} 1.0$ & IAA 1.0 & $6.79 \pm 0.48$ & $2.57 \pm 0.80^{\mathrm{b}}$ \\
3. & BAP 1.0 & $\mathrm{GA}_{3} 1.0$ & IAA 1.0 & $6.33 \pm 1.21$ & $2.08 \pm 0.21^{\mathrm{c}}$ \\
4. & BAP 1.0 & $\mathrm{GA}_{3} 0.5$ & IAA 1.0 & $5.50 \pm 1.04$ & $3.03 \pm 0.20^{\mathrm{a}}$ \\
5. & BAP 1.0 & $\mathrm{GA}_{3} 0.5$ & IAA 1.0 & $5.50 \pm 0.86$ & $3.01 \pm 0.15^{\mathrm{a}, \mathrm{b}}$ \\
\hline
\end{tabular}

Mean values within a column with the same letter are not significantly different at $p<0.05$. (Duncan's Multiple Range Test).

Primary explants failed to respond to MS medium without plant growth regulators, that is why this variant was withdrawn from our investigation. The hormonal investigation, regardless of the combinations and the concentration used, resulted in the response of explants $(100 \%)$ and gave the largest number of new cloned shoots, with the value between $5.50 \pm 0.86$ and $6.79 \pm 0.48$. The values of the mean number of shoots calculated per one explant were not significantly different regardless of the increase in concentration of BAP and $\mathrm{GA}_{3}$ in the culture medium, on which shoots grew (Figure 1; Table 1).

It is worth noticing that shoots grew vigorously, did not develop roots spontaneously, and also did not show any signs of verification or callusing at base, which is important for obtaining uniform shoot biomass. This study indicated the alternative method for effective and rapid shoot multiplication of $E$. alpinum. However, the increase in the concentration of BAP did not provide the highest biotechnological parameters compared to our previous studies [6,7]. In the case of E. planum, the highest mean number of shoots developed via axillary buds was 15.58 \pm 0.54-17.10 \pm 0.60 shoots per explant, depending on the culture media: MS + BAP $1.0 \mathrm{mg} / \mathrm{L}+\mathrm{IAA} 1.0 \mathrm{mg} / \mathrm{L}$ or MS + BAP $1.0 \mathrm{mg} / \mathrm{L}+\mathrm{IAA} 0.1 \mathrm{mg} / \mathrm{L}$ [14]. More shoots (13.30 \pm 3.73$)$, comparing to the control, were obtained for E. campestre when cultured on the same media composition as for E. planum [15]. The efficiency of shoot multiplication for $E$. maritimum varied between $1.2 \pm 0.20$ and $4.4 \pm 0.24$ shoots per explant on the different media variants. The highest value was observed for shoots growing on MS media supplemented with BAP $1.0 \mathrm{mg} / \mathrm{L}$ and IAA $0.1 \mathrm{mg} / \mathrm{L}[16]$. 
This technique aims to obtain a large number of homogeneous plants, using only a small fragment of the donor plant, in a relatively short time. Plant multiplication via axillary bud development, as used in our experiment, provides a renewable, inexhaustible amount of the raw material, allowing for the assessment of the phytochemical profile and testing the biological activity of the extracts, which is particularly important in the case of a rare and endangered plant. In addition, it is the alternative method of clonal multiplication of a plant from a different climate zone and of a low germination rate [8].

\subsection{The Phytochemical Analysis of Shoots from In Vitro Cultures and In Vivo Plants}

Shoots harvested from in vitro cultures as well as shoots from in vivo plants were subjected to the phytochemical analysis. The LC-MS base peak and the UV (270 and $330 \mathrm{~nm})$ chromatograms of the Eryngium alpinum L. are presented in Figures 2 and 3.

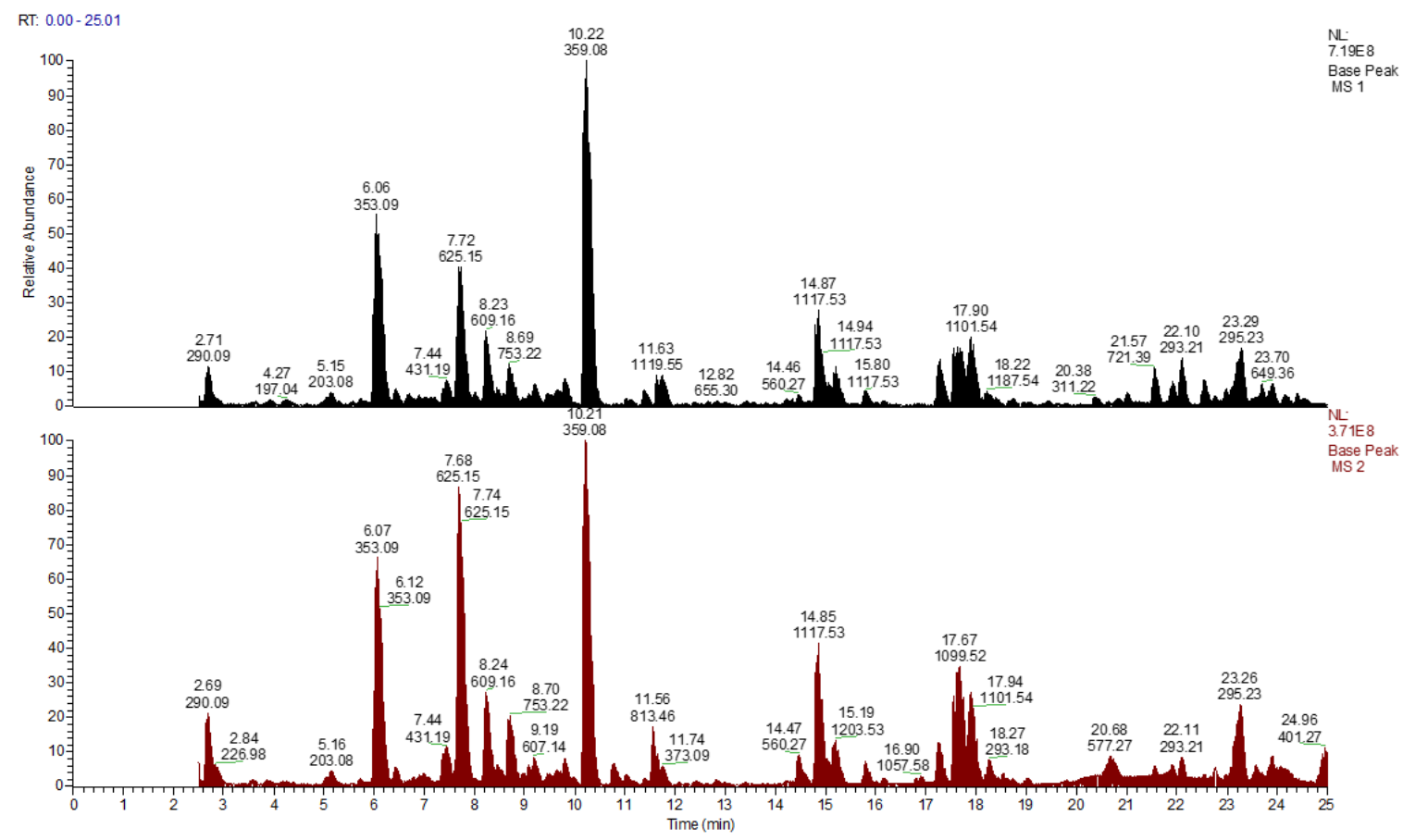

Figure 2. The qualitative analyses of the Eryngium alpinum L. samples from shoots of in vivo plants (black) and in vitro cultures (red). The base peak chromatograms in negative ion mode were obtained using the UPLC-HESI-II-HRMS system.

The retention times (RT), the observed and reference exact ion mass, the fragmentation spectra and the details are presented for the annotated compounds in Table 2. The annotation of compounds was carried out by comparing the observed and calculated exact mass for ions and the fragmentation pattern in positive and negative ion modes. Identification was complemented by applying the commercially available standards. During the analysis, 98 compounds were annotated and nine compounds were confirmed using the external standards. The main annotated compounds were phenylpropanoids, such as flavonoids (F), hydroxycinnamic acid derivates (HCA), and coumarins (C). Benzoic acid derivates (BA) and triterpenoid saponins (TT) were recognized in the samples. Other annotated groups of compounds were amino acids (AA), nucleotides (NA), carboxylic acids, some vitamins, and phytohormones. 
Table 2. The annotated compounds in the shoot extracts of Eryngium alpinum L. from in vitro culture and in vivo plant. Metabolites detected by UPLC-HESI-II-HRMS.

The compounds were characterized by the exact mass and the fragmentation pattern in negative and positive ion mode.

\begin{tabular}{|c|c|c|c|c|c|c|c|c|c|c|c|c|}
\hline No & RT [min] & Ion mode & $\begin{array}{l}\text { Observed } \\
m / z\end{array}$ & $\begin{array}{l}\text { Reference } \\
m / z\end{array}$ & $\begin{array}{r}\text { Delta } \\
(\text { ppm) }\end{array}$ & $\begin{array}{l}\text { Molecular } \\
\text { Formula }\end{array}$ & Fragmentation & Name & MSI Level $^{\text {a }}$ & Class $^{b}$ & $\mathrm{CID}^{\mathrm{c}}$ & Ref \\
\hline 1 & 2.58 & {$[\mathrm{M}+\mathrm{H}]^{+}$} & 175.11955 & 175.11896 & 3.4 & $\mathrm{C}_{6} \mathrm{H}_{14} \mathrm{~N}_{4} \mathrm{O}_{2}$ & $\begin{array}{c}60.0564,70.0660,116.0717,160.0981,158.0928 \\
158.0921\end{array}$ & Arginine & 3 & AA & 6322 & \\
\hline 2 & 2.7 & {$[\mathrm{M}-\mathrm{H}]^{-}$} & 191.0191 & 191.01973 & -3.3 & $\mathrm{C}_{6} \mathrm{H}_{8} \mathrm{O}_{7}$ & $57.0329,111.0069,129.0175,191.0193$ & Citric acid & $1^{\mathrm{s}}$ & CA & 311 & \\
\hline 3 & 2.7 & {$[\mathrm{M}-\mathrm{H}]^{-}$} & 243.06236 & 243.06226 & 0.4 & $\mathrm{C}_{9} \mathrm{H}_{12} \mathrm{~N}_{2} \mathrm{O}_{6}$ & $\begin{array}{l}82.0280,110.0228,122.0231,140.0340 \\
152.0331,200.0553 .\end{array}$ & Uridine & 2 & $\mathrm{~N}$ & 6029 & \\
\hline \multirow{2}{*}{4} & 2.7 & {$[\mathrm{M}-\mathrm{H}]^{-}$} & 180.0657 & 180.06662 & -5.1 & $\mathrm{C}_{9} \mathrm{H}_{11} \mathrm{NO}_{3}$ & 72.0073, 93.0335, 119.0484, 163.0383, 180.0649, & \multirow{2}{*}{ Tyrosine } & \multirow{2}{*}{$1^{\mathrm{s}}$} & \multirow{2}{*}{ AA } & \multirow{2}{*}{6057} & \multirow{2}{*}{3} \\
\hline & 2.7 & {$[\mathrm{M}+\mathrm{H}]^{+}$} & 182.0817 & 182.08118 & 2.9 & $\mathrm{C}_{9} \mathrm{H}_{11} \mathrm{NO}_{3}$ & $\begin{array}{c}119.0493,123.0442,136.0758,147.0441, \\
165.0553,182.0824\end{array}$ & & & & & \\
\hline 5 & 2.7 & {$[\mathrm{M}+\mathrm{H}]^{+}$} & 268.10495 & 268.10403 & 3.4 & $\mathrm{C}_{10} \mathrm{H}_{13} \mathrm{~N}_{5} \mathrm{O}_{4}$ & 57.0346, 136.0624, 268.1039, & Adenosine & 2 & $\mathrm{~N}$ & 60961 & \\
\hline 6 & 2.73 & {$[\mathrm{M}-\mathrm{H}]^{-}$} & 282.08435 & 282.08438 & -0.1 & $\mathrm{C}_{10} \mathrm{H}_{13} \mathrm{~N}_{5} \mathrm{O}_{5}$ & 133.0141, 150.0403, & Guanosine & 2 & $\mathrm{~N}$ & 135398635 & \\
\hline 7 & 2.93 & {$[\mathrm{M}+\mathrm{H}]^{+}$} & 276.14529 & 276.1445 & 2.9 & $\mathrm{C}_{12} \mathrm{H}_{23} \mathrm{NO}_{7}$ & $\begin{array}{c}86.0970,132.1028,212.1273,230.1401 \\
258.1352,276.1455\end{array}$ & $N$-Fructosyl isoleucine & 3 & AA & 137530247 & \\
\hline 8 & 3 & {$[\mathrm{M}-\mathrm{H}]^{-}$} & 182.0449 & 182.04588 & -5.4 & $\mathrm{C}_{8} \mathrm{H}_{9} \mathrm{NO}_{4}$ & $108.0445,120.0439,138.0539,182.0458$ & 4-Pyridoxic acid & 2 & $\mathrm{O}$ & 6723 & \\
\hline 9 & 3.65 & {$[\mathrm{M}+\mathrm{H}]^{+}$} & 192.06627 & 192.06552 & 3.9 & $\mathrm{C}_{10} \mathrm{H}_{9} \mathrm{NO}_{3}$ & $146.0598,192.1035$ & $\begin{array}{l}\text { 5-Hydroxy-3-indoleacetic } \\
\text { acid }\end{array}$ & 3 & $\mathrm{PH}$ & 1826 & \\
\hline 10 & 3.76 & {$[\mathrm{M}+\mathrm{H}]^{+}$} & 175.11923 & 175.11896 & 1.5 & $\mathrm{C}_{6} \mathrm{H}_{14} \mathrm{~N}_{4} \mathrm{O}_{2}$ & $60.0564,116.0717,160.0981,158.0928,158.0921$ & Arginine & 3 & AA & 6322 & \\
\hline 11 & 3.77 & {$[\mathrm{M}+\mathrm{H}]^{+}$} & 209.09282 & 209.09207 & 3.6 & $\mathrm{C}_{10} \mathrm{H}_{12} \mathrm{~N}_{2} \mathrm{O}_{3}$ & $\begin{array}{c}\text { 74.0249, 120.0449, 136.0759, 146.0599, } \\
163.0859,174.0547,192.0658\end{array}$ & Kynurenine & 2 & AA & 846 & \\
\hline 12 & 3.92 & {$[\mathrm{M}-\mathrm{H}]^{-}$} & 359.0995 & 359.0977 & 5 & $\mathrm{C}_{15} \mathrm{H}_{20} \mathrm{O}_{10}$ & - & Glucosyringic acid & 3 & BA & - & 4 \\
\hline 13 & 4.01 & {$[\mathrm{M}-\mathrm{H}]^{-}$} & 338.08862 & 338.08789 & 2.2 & $\mathrm{C}_{15} \mathrm{H}_{17} \mathrm{NO}_{8}$ & $132.0443,176.0334$ & $\begin{array}{c}\text { Indole }+1 O, 1 \text { carboxy } \\
\text { O-Hex; }\end{array}$ & 3 & AA & - & \\
\hline 14 & 4.17 & {$[\mathrm{M}+\mathrm{H}]^{+}$} & 213.07613 & 213.07574 & 1.8 & $\mathrm{C}_{10} \mathrm{H}_{12} \mathrm{O}_{5}$ & $149.0606,195.0643$ & Trimethoxybenzoic acid & 3 & BA & - & \\
\hline 15 & 4.21 & {$[\mathrm{M}-\mathrm{H}]^{-}$} & 315.0725 & 315.071 & 4.8 & $\mathrm{C}_{13} \mathrm{H}_{16} \mathrm{O}_{9}$ & $108.0199,152.0102,315.0696$ & $\begin{array}{l}\text { Dihydroxybenzoic acid } \\
\text { hexoside }\end{array}$ & 3 & BA & - & 4 \\
\hline 16 & 4.26 & {$[\mathrm{M}-\mathrm{H}]^{-}$} & 167.035 & 167.03499 & 0.1 & $\mathrm{C}_{8} \mathrm{H}_{8} \mathrm{O}_{4}$ & $108.0200,123.0434,152.0102,167.0343$ & Vanillic acid & 2 & BA & 8468 & \\
\hline 17 & 4.26 & {$[\mathrm{M}+\mathrm{H}]^{+}$} & 179.03462 & 179.03389 & 4.1 & $\mathrm{C}_{9} \mathrm{H}_{6} \mathrm{O}_{4}$ & $\begin{array}{c}105.6870,107.0502,123.0445,133.0281, \\
151.0394,179.1061\end{array}$ & Dihydroxycoumarin & 2 & C & - & \\
\hline 18 & 4.26 & {$[\mathrm{M}-\mathrm{H}]^{-}$} & 329.08838 & 329.08841 & -0.1 & $\mathrm{C}_{14} \mathrm{H}_{18} \mathrm{O}_{9}$ & $108.0200,123.0434,152.0102,167.0343$ & Vanillic acid hexoside & 3 & BA & - & \\
\hline 19 & 4.39 & {$[\mathrm{M}-\mathrm{H}]^{-}$} & 218.1031 & 218.10339 & -1.3 & $\mathrm{C}_{9} \mathrm{H}_{17} \mathrm{NO}_{5}$ & $71.0121,88.0389,99.0432,116.0707,140.2067$ & Pantothenic acid & 3 & $\mathrm{O}$ & 6613 & \\
\hline 20 & 4.95 & {$[\mathrm{M}]^{+}$} & 266.1389 & 266.13812 & 2.9 & $\mathrm{C}_{14} \mathrm{H}_{20} \mathrm{NO}_{4}$ & $95.0861,163.0382,207.0648$ & Caffeoylcholine & 2 & $\mathrm{HC}$ & 6440794 & \\
\hline \multirow{2}{*}{21} & 5.16 & {$[\mathrm{M}-\mathrm{H}]^{-}$} & 203.08162 & 203.0826 & -4.8 & $\mathrm{C}_{11} \mathrm{H}_{12} \mathrm{~N}_{2} \mathrm{O}_{2}$ & 116.0494, 142.0644, 159.0909, 186.0549, & \multirow{2}{*}{ Tryptophan } & \multirow{2}{*}{$1^{\mathrm{s}}$} & \multirow{2}{*}{ AA } & \multirow{2}{*}{6305} & \multirow{2}{*}{3} \\
\hline & 5.16 & {$[\mathrm{M}+\mathrm{H}]^{+}$} & 205.09726 & 205.09715 & 0.5 & $\mathrm{C}_{11} \mathrm{H}_{12} \mathrm{~N}_{2} \mathrm{O}_{2}$ & $\begin{array}{c}118.0661,132.0812,146.0598,159.0912 \\
170.0601,188.0714\end{array}$ & & & & & \\
\hline
\end{tabular}


Table 2. Cont

\begin{tabular}{|c|c|c|c|c|c|c|c|c|c|c|c|c|}
\hline No & RT [min] & Ion mode & $\begin{array}{l}\text { Observed } \\
m / z\end{array}$ & $\begin{array}{l}\text { Reference } \\
\mathrm{m} / \mathrm{z}\end{array}$ & $\begin{array}{l}\text { Delta } \\
(\mathrm{ppm})\end{array}$ & $\begin{array}{l}\text { Molecular } \\
\text { Formula }\end{array}$ & Fragmentation & Name & MSI Level $^{\text {a }}$ & Class $^{b}$ & CID $^{c}$ & Ref. \\
\hline 22 & 5.18 & {$[\mathrm{M}]^{+}$} & 250.1441 & 250.14322 & 3.5 & $\mathrm{C}_{14} \mathrm{H}_{20} \mathrm{NO}_{3}$ & - & Coumaroylcholine & 2 & $\mathrm{HC}$ & 6440550 & \\
\hline 23 & 5.3 & {$[\mathrm{M}-\mathrm{H}]^{-}$} & 181.05003 & 181.05063 & -3.3 & $\mathrm{C}_{9} \mathrm{H}_{10} \mathrm{O}_{4}$ & 72.9914, 119.0483, 135.0436, 163.0382, 181.0498 & $\begin{array}{l}\text { Hydroxyphenyllactic } \\
\text { acid }\end{array}$ & 3 & BA & - & \\
\hline 24 & 5.42 & {$[\mathrm{M}-\mathrm{H}]^{-}$} & 339.07239 & 339.07214 & 0.7 & $\mathrm{C}_{15} \mathrm{H}_{16} \mathrm{O}_{9}$ & 177.0192, & Esculin & 2 & $\mathrm{C}$ & 5281417 & \\
\hline 25 & 5.57 & {$[\mathrm{M}-\mathrm{H}]^{-}$} & 353.08743 & 353.0878 & -1 & $\mathrm{C}_{16} \mathrm{H}_{18} \mathrm{O}_{9}$ & 161.0231, 173.0437, 179.0333, 191.0195, & Neochlorogenic acid & $1^{\mathrm{s}}$ & $\mathrm{HC}$ & 5280633 & 1,3 \\
\hline 26 & 5.58 & {$[\mathrm{M}-\mathrm{H}]^{-}$} & 355.0672 & 355.06561 & 4.5 & $\mathrm{C}_{15} \mathrm{H}_{16} \mathrm{O}_{10}$ & $\begin{array}{c}135.0794,147.0285,163.0382,179.0697, \\
191.0199,209.0293\end{array}$ & Coumaroyl $+\mathrm{C}_{6} \mathrm{H}_{9} \mathrm{O}_{8}$ & 3 & $\mathrm{HC}$ & - & \\
\hline 27 & 5.7 & {$[\mathrm{M}+\mathrm{H}]^{+}$} & 167.07097 & 167.07027 & 4.2 & $\mathrm{C}_{9} \mathrm{H}_{10} \mathrm{O}_{3}$ & $111.0448,139.0757,149.0232,167.0708$ & Dimethoxybenzaldehyde & 3 & $\mathrm{BA}$ & - & \\
\hline 28 & 5.71 & {$[\mathrm{M}+\mathrm{H}]^{+}$} & 261.14514 & 261.1445 & 2.5 & $\mathrm{C}_{11} \mathrm{H}_{20} \mathrm{~N}_{2} \mathrm{O}_{5}$ & $\begin{array}{c}84.0451,86.0605,132.1017,198.1126,244.1189 \\
261.1274\end{array}$ & Glutamylleucine & 2 & AA & 9856500 & \\
\hline 29 & 5.73 & {$[\mathrm{M}+\mathrm{H}]^{+}$} & 517.15778 & 517.15574 & 4 & $\mathrm{C}_{22} \mathrm{H}_{28} \mathrm{O}_{14}$ & $193.0489,178.0266,165.0556,133.0285$ & $\begin{array}{l}\text { Scopoletin } \\
\text { 7-O-dihexoside }\end{array}$ & 2 & C & - & \\
\hline 30 & 5.91 & {$[\mathrm{M}-\mathrm{H}]^{-}$} & 341.08795 & 341.08726 & 2 & $\mathrm{C}_{15} \mathrm{H}_{18} \mathrm{O}_{9}$ & 119.0336, 161.0236, 179.0339, & Caffeic acid glucoside & 2 & $\mathrm{HC}$ & 5281761 & 3 \\
\hline \multirow{2}{*}{31} & 6.06 & {$[\mathrm{M}+\mathrm{H}]^{+}$} & 355.1022 & 355.10236 & -0.5 & $\mathrm{C}_{16} \mathrm{H}_{18} \mathrm{O}_{9}$ & 163.0385, & \multirow{2}{*}{ Chlorogenic acid } & \multirow{2}{*}{$1^{\mathrm{s}}$} & \multirow{2}{*}{$\mathrm{HC}$} & \multirow{2}{*}{1794427} & \multirow{2}{*}{3} \\
\hline & 6.06 & {$[\mathrm{M}-\mathrm{H}]^{-}$} & 353.08755 & 353.0878 & -0.7 & $\mathrm{C}_{16} \mathrm{H}_{18} \mathrm{O}_{9}$ & $135.0437,161.0226,179.0334,191.0546$ & & & & & \\
\hline 32 & 6.17 & {$[\mathrm{M}+\mathrm{H}]^{+}$} & 295.12949 & 295.12949 & 0 & $\mathrm{C}_{14} \mathrm{H}_{18} \mathrm{~N}_{2} \mathrm{O}_{5}$ & $120.0811,166.0859,186.0907,232.0963$ & Glutamylphenylalanine & 2 & AA & 111299 & \\
\hline 33 & 6.3 & $\begin{array}{c}{[\mathrm{M}-\mathrm{H}]^{-}} \\
]^{-}\end{array}$ & 399.09326 & 399.09299 & 0.7 & $\mathrm{C}_{16} \mathrm{H}_{18} \mathrm{O}_{9}$ & 135.0437, 176.0116, 191.0346, 221.0073 & Scopolin & 2 & C & 439514 & \\
\hline 34 & 6.49 & {$[\mathrm{M}-\mathrm{H}]^{-}$} & 367.1038 & 367.10199 & 4.9 & $\mathrm{C}_{17} \mathrm{H}_{20} \mathrm{O}_{9}$ & $\begin{array}{c}134.0357,149.0238,163.0483,191.0562, \\
193.0493\end{array}$ & Feruloylquinic acid & 3 & $\mathrm{HC}$ & 10177048 & 3,4 \\
\hline 35 & 6.5 & {$[\mathrm{M}-\mathrm{H}]^{-}$} & 355.0665 & 355.06509 & 4 & $\mathrm{C}_{15} \mathrm{H}_{16} \mathrm{O}_{10}$ & $147.0282,191.0194,209.0293$ & Coumaroyl $+\mathrm{C}_{6} \mathrm{H}_{9} \mathrm{O}_{8}$ & 3 & $\mathrm{HC}$ & - & \\
\hline 36 & 6.77 & {$[\mathrm{M}+\mathrm{H}]^{+}$} & 223.06041 & 223.0601 & 1.4 & $\mathrm{C}_{11} \mathrm{H}_{10} \mathrm{O}_{5}$ & $\begin{array}{l}\text { 149.0244, 162.0302, 177.0907, 190.0266, } \\
\text { 207.0280, }\end{array}$ & Isofraxidin & 2 & C & 5318565 & \\
\hline \multirow{2}{*}{37} & \multirow{2}{*}{7.00} & {$[\mathrm{M}+\mathrm{H}]^{+}$} & 773.21649 & 773.2135 & 3.9 & \multirow{2}{*}{$\mathrm{C}_{33} \mathrm{H}_{40} \mathrm{O}_{21}$} & $132.1144,228.9691,303.0504$ & \multirow{2}{*}{$\begin{array}{l}\text { Quercetin-3-O-dihexoside-7- } \\
\text { O-rhamnoside }\end{array}$} & \multirow{2}{*}{3} & \multirow{2}{*}{$\mathrm{F}$} & \multirow{2}{*}{57393076} & \\
\hline & & {$[\mathrm{M}-\mathrm{H}]^{-}$} & 771.20292 & 771.19839 & 5.9 & & $\begin{array}{c}151.0028,178.9972,300.0273,446.0863, \\
625.1580\end{array}$ & & & & & \\
\hline \multirow[t]{2}{*}{38} & \multirow[t]{2}{*}{7.02} & {$[\mathrm{M}-\mathrm{H}]^{-}$} & 353.08746 & 353.08621 & 3.5 & \multirow{2}{*}{$\mathrm{C}_{16} \mathrm{H}_{18} \mathrm{O}_{9}$} & $135.0437,161.0226,179.0334,191.0546(100 \%)$ & \multirow{2}{*}{$\begin{array}{l}\text { Caffeoylquinic acid } \\
\text { (Isochlorogenic acid) }\end{array}$} & \multirow{2}{*}{2} & \multirow[t]{2}{*}{$\mathrm{HC}$} & \multirow{2}{*}{5315832} & \multirow{2}{*}{1} \\
\hline & & {$[\mathrm{M}+\mathrm{H}]^{+}$} & 355.10339 & 355.10236 & 2.9 & & $137.0612,163.0386,201.0543$ & & & & & \\
\hline 39 & 7.14 & {$[\mathrm{M}-\mathrm{H}]^{-}$} & 337.09293 & 337.09219 & 2.2 & $\mathrm{C}_{16} \mathrm{H}_{18} \mathrm{O}_{8}$ & $163.0483,191.0562$ & Coumaroylquinic acid & 2 & $\mathrm{HC}$ & 6441280 & 3 \\
\hline \multirow{2}{*}{40} & \multirow[t]{2}{*}{7.41} & {$[\mathrm{M}+\mathrm{H}]^{+}$} & 611.16309 & 611.16121 & 3.1 & \multirow[t]{2}{*}{$\mathrm{C}_{27} \mathrm{H}_{30} \mathrm{O}_{16}$} & 303.0502 & \multirow{2}{*}{$\begin{array}{c}\text { Quercetin-3- } \\
\text { O-hexoside-7-O-rhamnoside }\end{array}$} & \multirow[t]{2}{*}{2} & \multirow[t]{2}{*}{$\mathrm{F}$} & \multirow[t]{2}{*}{25080064} & \multirow[t]{2}{*}{2} \\
\hline & & {$[\mathrm{M}-\mathrm{H}]^{-}$} & 609.15906 & 609.15612 & 4.8 & & $299.0215,301.0368,447.0932,463.0879$ & & & & & \\
\hline 41 & 7.49 & {$[\mathrm{M}-\mathrm{H}]^{-}$} & 345.13425 & 345.13437 & -0.3 & $\mathrm{C}_{19} \mathrm{H}_{22} \mathrm{O}_{6}$ & $143.0849,221.1323,239.1435,273.1489$ & Gibberellic acid & 3 & $\mathrm{O}$ & 6466 & \\
\hline
\end{tabular}


Table 2. Cont.

\begin{tabular}{|c|c|c|c|c|c|c|c|c|c|c|c|c|}
\hline No & RT [min] & Ion mode & $\begin{array}{c}\text { Observed } \\
\mathrm{m} / \mathrm{z}\end{array}$ & $\begin{array}{l}\text { Reference } \\
\mathrm{m} / \mathrm{z}\end{array}$ & $\begin{array}{l}\text { Delta } \\
\text { (ppm) }\end{array}$ & $\begin{array}{l}\text { Molecular } \\
\text { Formula }\end{array}$ & Fragmentation & Name & MSI Level ${ }^{a}$ & Class $^{b}$ & $\operatorname{CID}^{\mathrm{c}}$ & Ref. \\
\hline \multirow[t]{2}{*}{42} & \multirow[t]{2}{*}{7.51} & {$[\mathrm{M}-\mathrm{H}]^{-}$} & 755.2341 & 755.23986 & -7.6 & \multirow[t]{2}{*}{$\mathrm{C}_{33} \mathrm{H}_{40} \mathrm{O}_{20}$} & $\begin{array}{c}176.8649,227.0346,255.0293,284.0326 \\
285.0404,609.1585\end{array}$ & \multirow{2}{*}{$\begin{array}{l}\text { Kaempferol-O } \\
\text {-rhamnodihexoside }\end{array}$} & \multirow[t]{2}{*}{3} & \multirow[t]{2}{*}{$\mathrm{F}$} & \multirow[t]{2}{*}{-} & \\
\hline & & {$[\mathrm{M}+\mathrm{H}]^{+}$} & 757.2229 & 757.21913 & 5.0 & & 287.0552 & & & & & \\
\hline \multirow[t]{2}{*}{43} & 7.71 & {$[\mathrm{M}-\mathrm{H}]^{-}$} & 625.14482 & 625.14105 & 6 & $\mathrm{C}_{27} \mathrm{H}_{30} \mathrm{O}_{17}$ & $\begin{array}{c}151.0014,178.9977,255.0304,271.0252 \\
300.0289,301.0323\end{array}$ & \multirow[t]{2}{*}{ Quercetin-3-O-dihexoside } & \multirow[t]{2}{*}{2} & \multirow[t]{2}{*}{$\mathrm{F}$} & \multirow[t]{2}{*}{14185727} & \multirow[t]{2}{*}{4} \\
\hline & 7.71 & {$[\mathrm{M}+\mathrm{H}]^{+}$} & 627.15741 & 627.15558 & 2.9 & $\mathrm{C}_{27} \mathrm{H}_{30} \mathrm{O}_{17}$ & $159.8611,281.4450,303.0503$ & & & & & \\
\hline \multirow[t]{2}{*}{44} & 7.74 & {$[\mathrm{M}+\mathrm{H}]^{+}$} & 449.10812 & 449.10785 & 0.6 & $\mathrm{C}_{21} \mathrm{H}_{20} \mathrm{O}_{11}$ & $\begin{array}{c}130.1475,287.0541,299.0568,329.0639 \\
353.0654\end{array}$ & \multirow[t]{2}{*}{ Luteolin-C-hexoside } & \multirow[t]{2}{*}{3} & \multirow[t]{2}{*}{$\mathrm{F}$} & \multirow[t]{2}{*}{ - } & \\
\hline & 7.74 & {$[\mathrm{M}-\mathrm{H}]^{-}$} & 447.09238 & 447.09329 & -2 & $\mathrm{C}_{21} \mathrm{H}_{20} \mathrm{O}_{11}$ & $285.0380,297.0394,327.0507,357.0601$ & & & & & \\
\hline \multirow{2}{*}{45} & \multirow{2}{*}{7.76} & {$[\mathrm{M}-\mathrm{H}]^{-}$} & 367.1038 & 367.10291 & 2.4 & \multirow{2}{*}{$\mathrm{C}_{17} \mathrm{H}_{20} \mathrm{O}_{9}$} & $134.0357,173.0386,191.0546$ & \multirow{2}{*}{ Feruloylquinic acid } & \multirow{2}{*}{3} & \multirow{2}{*}{$\mathrm{HC}$} & \multirow{2}{*}{10177048} & \multirow{2}{*}{3,4} \\
\hline & & {$[\mathrm{M}+\mathrm{H}]^{+}$} & 369.11871 & 369.11801 & 1.9 & & $\begin{array}{c}\text { 117.0337, } 145.0289,149.0607,163.0385 \\
177.0550,195.0642\end{array}$ & & & & & \\
\hline \multirow{2}{*}{46} & \multirow[t]{2}{*}{7.86} & {$[\mathrm{M}+\mathrm{H}]^{+}$} & 755.20820 & 755.20660 & 4.2 & \multirow{2}{*}{$\mathrm{C}_{33} \mathrm{H}_{38} \mathrm{O}_{20}$} & $127.0398,145.0505,303.0504$ & \multirow{2}{*}{ Unknown flavonoid } & \multirow[t]{2}{*}{3} & $\mathrm{~F}$ & - & \\
\hline & & $\left.[\mathrm{M}-\mathrm{H}]^{-}\right]^{-}$ & 753.18783 & 753.19064 & -3.7 & & $301.0349,446.0853,463.0864$ & & & & & \\
\hline 47 & 8.03 & {$[\mathrm{M}+\mathrm{H}]^{+}$} & 227.12856 & 227.12779 & 3.4 & $\mathrm{C}_{12} \mathrm{H}_{18} \mathrm{O}_{4}$ & $\begin{array}{c}, 131.0861,149.0969,167.1077,191.1070 \\
\text { 209.1177, 227.1267 }\end{array}$ & $\begin{array}{l}\text { 12-Hydroxyjasmonic } \\
\text { acid }\end{array}$ & 2 & $\mathrm{O}$ & 5497122 & \\
\hline 48 & 8.17 & {$[\mathrm{M}-\mathrm{H}]^{-}$} & 172.09709 & 172.09792 & -4.8 & $\mathrm{C}_{8} \mathrm{H}_{15} \mathrm{NO}_{3}$ & $130.0862,172.0976$ & $N$-Acetylleucine & 2 & AA & 70912 & \\
\hline 49 & 8.25 & {$[\mathrm{M}-\mathrm{H}]^{-}$} & 609.14894 & 609.14612 & 4.6 & $\mathrm{C}_{27} \mathrm{H}_{30} \mathrm{O}_{16}$ & $\begin{array}{l}\text { 151.0018, 163.0026, 178.9970, 227.0341, } \\
255.0299,284.0318,285.0379, \text { 609.1639 }\end{array}$ & Kaempferol-3-O-dihexoside & 2 & $\mathrm{~F}$ & 5282155 & 4 \\
\hline & & {$[\mathrm{M}+\mathrm{H}]^{+}$} & 611.16367 & 611.16064 & 5 & & $127.0398,287.0541$ & & & & & \\
\hline 50 & 8.4 & {$[\mathrm{M}-\mathrm{H}]^{-}$} & 192.066 & 192.06662 & -3.2 & $\mathrm{C}_{10} \mathrm{H}_{11} \mathrm{NO}_{3}$ & - & Phenylacetylglycine & 2 & AA & 68144 & \\
\hline 51 & 8.43 & $\left.[\mathrm{M}-\mathrm{H}]^{-}\right]^{-}$ & 367.1038 & 367.10199 & 4.9 & $\mathrm{C}_{17} \mathrm{H}_{20} \mathrm{O}_{9}$ & 191.0546 & Feruloylquinic acid & 3 & $\mathrm{HC}$ & 10177048 & \\
\hline 52 & 8.47 & {$[\mathrm{M}-\mathrm{H}]^{-}$} & 609.14887 & 609.14612 & 4.5 & $\mathrm{C}_{27} \mathrm{H}_{30} \mathrm{O}_{16}$ & $\begin{array}{c}\text { 133.8210, } 151.0018,177.9567,255.0308 \\
271.0236,285.0425,300.0290,301.0323 \\
609.1639\end{array}$ & Quercetin-3-O-rutinoside & $1^{\mathrm{s}}$ & $\mathrm{F}$ & 5280805 & 1 \\
\hline & & {$[\mathrm{M}+\mathrm{H}]^{+}$} & 611.16321 & 611.16122 & 3.3 & & $129.0572,287.0541,303.0504$ & & & & & \\
\hline 53 & 8.67 & {$[\mathrm{M}-\mathrm{H}]^{-}$} & 463.08749 & 463.0882 & -1.5 & $\mathrm{C}_{21} \mathrm{H}_{20} \mathrm{O}_{12}$ & $\begin{array}{c}151.0036,255.0304,271.0255,287.2002 \\
300.0289,301.0315\end{array}$ & Quercetin-3-O-galactoside & $1^{\mathrm{s}}$ & $\mathrm{F}$ & 5281643 & \\
\hline & & {$[\mathrm{M}+\mathrm{H}]^{+}$} & 465.10483 & 465.10275 & 4.5 & & $142.7517,257.0451,285.0396,303.0503$ & & & & & \\
\hline 54 & 8.67 & {$[\mathrm{M}-\mathrm{H}]^{-}$} & 593.15129 & 593.15118 & 0.2 & $\mathrm{C}_{27} \mathrm{H}_{30} \mathrm{O}_{15}$ & $\begin{array}{c}227.0337,255.0302,272.9905,284.0320 \\
285.0383,593.1517\end{array}$ & Kaempferol-3-O-rutinoside & 2 & $\mathrm{~F}$ & 5318767 & 2 \\
\hline & & {$[\mathrm{M}+\mathrm{H}]^{+}$} & 595.16882 & 595.16577 & 5.1 & & $85.0296,164.3077,253.8810,287.0542$ & & & & & \\
\hline 55 & 8.67 & {$[\mathrm{M}-\mathrm{H}]^{-}$} & 313.09305 & 313.09235 & 2.3 & $\mathrm{C}_{14} \mathrm{H}_{18} \mathrm{O}_{8}$ & 121.0280 & Glucovanillin & 3 & BA & 6452133 & \\
\hline
\end{tabular}


Table 2. Cont.

\begin{tabular}{|c|c|c|c|c|c|c|c|c|c|c|c|c|}
\hline No & RT [min] & Ion mode & $\begin{array}{l}\text { Observed } \\
\mathrm{m} / \mathrm{z}\end{array}$ & $\begin{array}{l}\text { Reference } \\
\mathrm{m} / \mathrm{z}\end{array}$ & $\begin{array}{l}\text { Delta } \\
\text { (ppm) }\end{array}$ & $\begin{array}{l}\text { Molecular } \\
\text { Formula }\end{array}$ & Fragmentation & Name & MSI Level $^{\text {a }}$ & Class $^{b}$ & $\mathrm{CID}^{\mathrm{c}}$ & Ref. \\
\hline 56 & 8.68 & {$[\mathrm{M}+\mathrm{H}]^{+}$} & 609.14679 & 609.14557 & 2.0 & $\mathrm{C}_{27} \mathrm{H}_{28} \mathrm{O}_{16}$ & 303.0501 & Unknown & 3 & F & - & \\
\hline \multirow[t]{2}{*}{57} & \multirow[t]{2}{*}{8.7} & {$[\mathrm{M}-\mathrm{H}]^{-}$} & 753.21992 & 753.22421 & -5.7 & \multirow[t]{2}{*}{$\mathrm{C}_{33} \mathrm{H}_{38} \mathrm{O}_{20}$} & $\begin{array}{c}151.0018,255.0304,271.0245,300.0290 \\
301.0324609 .1639\end{array}$ & \multirow[t]{2}{*}{ Unknown flavonoids } & \multirow[t]{2}{*}{3} & \multirow[t]{2}{*}{$\mathrm{F}$} & \multirow[t]{2}{*}{11498684} & \\
\hline & & {$[\mathrm{M}+\mathrm{H}]^{+}$} & 755.20740 & 755.20347 & 5.2 & & $179.3386,303.0505$ & & & & & \\
\hline \multirow[t]{2}{*}{58} & \multirow[t]{2}{*}{8.77} & {$[\mathrm{M}-\mathrm{H}]^{-}$} & 463.08752 & 463.0882 & -1.5 & \multirow[t]{2}{*}{$\mathrm{C}_{21} \mathrm{H}_{20} \mathrm{O}_{12}$} & $\begin{array}{c}151.0020,255.0296,271.0252,300.0288 \\
301.0371,463.0862\end{array}$ & \multirow[t]{2}{*}{ Quercetin-3-O-glucoside } & \multirow[t]{2}{*}{$1^{\mathrm{s}}$} & \multirow[t]{2}{*}{$\mathrm{F}$} & \multirow[t]{2}{*}{5280804} & \multirow[t]{2}{*}{1,4} \\
\hline & & {$[\mathrm{M}+\mathrm{H}]^{+}$} & 465.10287 & 465.10275 & 0.3 & & $\begin{array}{c}257.0451,275.6729,285.0396,303.0504 \\
465.1721\end{array}$ & & & & & \\
\hline 59 & 8.96 & {$[\mathrm{M}-\mathrm{H}]^{-}$} & 447.09442 & 447.09329 & 2.5 & $\mathrm{C}_{21} \mathrm{H}_{20} \mathrm{O}_{11}$ & $\begin{array}{c}167.4627,279.2310,284.0321,285.0379 \\
447.0933\end{array}$ & Luteolin 7-O-glucoside & 3 & $\mathrm{~F}$ & 5280637 & 2 \\
\hline 60 & 9.01 & {$[\mathrm{M}-\mathrm{H}]^{-}$} & 245.09334 & 245.09317 & 0.7 & $\mathrm{C}_{13} \mathrm{H}_{14} \mathrm{~N}_{2} \mathrm{O}_{3}$ & - & $\mathrm{N}$-Acetyltryptophan & 3 & AA & 2002 & \\
\hline 61 & 9.02 & {$[\mathrm{M}-\mathrm{H}]^{-}$} & 521.13037 & 521.12952 & 1.6 & $\mathrm{C}_{24} \mathrm{H}_{26} \mathrm{O}_{13}$ & $135.0531,161.0233,179.0336,197.0438$ & $\begin{array}{l}\text { Rosmarinic acid } \\
\text { glucoside }\end{array}$ & 2 & $\mathrm{HC}$ & 132604855 & 3 \\
\hline 62 & 9.12 & {$[\mathrm{M}+\mathrm{H}]^{+}$} & 223.06041 & 223.0601 & 1.4 & $\mathrm{C}_{11} \mathrm{H}_{10} \mathrm{O}_{5}$ & $\begin{array}{c}121.0291,149.0244,162.0302,177.0907 \\
190.0266,207.0280\end{array}$ & Fraxidin & 2 & C & 3083616 & \\
\hline \multirow{2}{*}{63} & \multirow{2}{*}{9.19} & {$[\mathrm{M}+\mathrm{H}]^{+}$} & 609.14673 & 609.14556 & 1.9 & \multirow{2}{*}{$\mathrm{C}_{27} \mathrm{H}_{28} \mathrm{O}_{16}$} & 303.0503 & \multirow{2}{*}{ Unknown flavonoids } & \multirow{2}{*}{3} & \multirow{2}{*}{$\mathrm{F}$} & \multirow{2}{*}{-} & \\
\hline & & {$[\mathrm{M}-\mathrm{H}]^{-}$} & 607.13054 & 607.12991 & -1.0 & & $\begin{array}{c}151.0025,178.9976,255.0293,271.0252 \\
300.0273,463.0867\end{array}$ & & & & & \\
\hline \multirow{2}{*}{64} & \multirow{2}{*}{9.19} & {$[\mathrm{M}+\mathrm{H}]^{+}$} & 739.21136 & 739.20856 & 3.8 & \multirow{2}{*}{$\mathrm{C}_{33} \mathrm{H}_{38} \mathrm{O}_{19}$} & 287.0552 & \multirow{2}{*}{ Unknown flavonoids } & \multirow{2}{*}{3} & \multirow{2}{*}{$\mathrm{F}$} & \multirow[t]{2}{*}{ - } & \\
\hline & & {$[\mathrm{M}-\mathrm{H}]^{-}$} & 737.20084 & 737.19291 & 9.8 & & $151.0023,255.0289,284.0325,285.0395$ & & & & & \\
\hline 65 & 9.23 & {$[\mathrm{M}-\mathrm{H}]^{-}$} & 505.09988 & 505.09875 & 2.2 & $\mathrm{C}_{23} \mathrm{H}_{22} \mathrm{O}_{13}$ & $151.0018,271.0246,300.0292,301.0322$ & $\begin{array}{c}\text { Quercetin } \\
\text { 3-(6-O-acetyl)-hexoside }\end{array}$ & 2 & $\mathrm{~F}$ & 10006384 & \\
\hline \multirow[t]{2}{*}{66} & \multirow[t]{2}{*}{9.25} & {$[\mathrm{M}-\mathrm{H}]^{-}$} & 593.15141 & 593.15118 & 0.4 & $\mathrm{C}_{27} \mathrm{H}_{30} \mathrm{O}_{15}$ & $\begin{array}{c}133.0971,151.0018,255.0304,284.0320 \\
285.0382\end{array}$ & $\begin{array}{l}\text { Luteolin-7-O- } \\
\text { ramnohexoside }\end{array}$ & 2 & $\mathrm{~F}$ & 5318767 & 2 \\
\hline & & {$[\mathrm{M}+\mathrm{H}]^{+}$} & 595.16858 & 595.16630 & 2.9 & & 287.0552 & & & & & \\
\hline 67 & 9.29 & {$[\mathrm{M}-\mathrm{H}]^{-}$} & 449.10779 & 449.10730 & 1.1 & $\mathrm{C}_{21} \mathrm{H}_{20} \mathrm{O}_{11}$ & 287.0544 & Kaempferol-O-hexoside I & 2 & $\mathrm{~F}$ & 5282149 & 2 \\
\hline & & & 447.09445 & 447.09329 & 2.6 & & $\begin{array}{c}150.1186,196.2700,227.0370,255.0304 \\
284.0321,285.0380\end{array}$ & & & & & \\
\hline 68 & 9.55 & {$[\mathrm{M}-\mathrm{H}]^{-}$} & 515.12018 & 515.11951 & 1.3 & $\mathrm{C}_{25} \mathrm{H}_{24} \mathrm{O}_{12}$ & $\begin{array}{c}135.0437,161.0230,179.0334,191.0546 \\
353.0867\end{array}$ & Dicaffeoylquinic acid & 3 & $\mathrm{HC}$ & 13604687 & 3 \\
\hline & & {$[\mathrm{M}+\mathrm{H}]^{+}$} & 517.13562 & 517.13403 & 3.1 & & $135.0442,145.0291,163.0386$ & & & & & \\
\hline 69 & 956 & {$[\mathrm{M}-\mathrm{H}]^{-}$} & 577.16005 & 577.15625 & 6.6 & $\mathrm{C}_{27} \mathrm{H}_{30} \mathrm{O}_{14}$ & 269.0468 & Apigenin-7-O- & 2 & $\mathrm{~F}$ & 5282150 & \\
\hline & & {$[\mathrm{M}+\mathrm{H}]^{+}$} & 579.17301 & 579.17084 & 3.7 & & $85.0294,200.8167,271.0601$ & rhamnohexoside & & & & \\
\hline
\end{tabular}


Table 2. Cont.

\begin{tabular}{|c|c|c|c|c|c|c|c|c|c|c|c|c|}
\hline No & RT [min] & Ion mode & $\begin{array}{c}\text { Observed } \\
\mathrm{m} / \mathrm{z}\end{array}$ & $\begin{array}{l}\text { Reference } \\
\mathrm{m} / \mathrm{z}\end{array}$ & $\begin{array}{r}\text { Delta } \\
(\mathrm{ppm})\end{array}$ & $\begin{array}{l}\text { Molecular } \\
\text { Formula }\end{array}$ & Fragmentation & Name & MSI Level a & Class $^{b}$ & $\mathrm{CID}^{\mathrm{c}}$ & Ref. \\
\hline \multirow[t]{2}{*}{70} & \multirow[t]{2}{*}{9.67} & {$[\mathrm{M}-\mathrm{H}]^{-}$} & 447.09457 & 447.09329 & 2.9 & \multirow[t]{2}{*}{$\mathrm{C}_{21} \mathrm{H}_{20} \mathrm{O}_{11}$} & $\begin{array}{c}227.0337,249.0604,255.0309,279.2349, \\
284.0317,285.0379\end{array}$ & \multirow{2}{*}{$\begin{array}{l}\text { Kaempferol-O-hexoside } \\
\text { II }\end{array}$} & \multirow[t]{2}{*}{2} & \multirow[t]{2}{*}{$\mathrm{F}$} & \multirow[t]{2}{*}{5282102} & \multirow[t]{2}{*}{2} \\
\hline & & {$[\mathrm{M}-\mathrm{H}]^{-}$} & 447.0943 & 447.09329 & 2.3 & & $279.2305,285.0385$ & & & & & \\
\hline 71 & 10.03 & {$[\mathrm{M}-\mathrm{H}]^{-}$} & 535.10642 & 535.10879 & -4.4 & $\mathrm{C}_{24} \mathrm{H}_{24} \mathrm{O}_{14}$ & $135.0436,179.0335,197.0448$ & $\begin{array}{l}\text { Rosmarinic acid, } \\
\text { glucuronide }\end{array}$ & 2 & $\mathrm{HC}$ & - & 3 \\
\hline 72 & 10.07 & {$[\mathrm{M}-\mathrm{H}]^{-}$} & 351.0724 & 351.07214 & 0.7 & $\mathrm{C}_{16} \mathrm{H}_{16} \mathrm{O}_{9}$ & - & $\begin{array}{l}\text { 4-Methylumbelliferyl } \\
\text { glucuronide }\end{array}$ & 3 & C & 91553 & \\
\hline 73 & 10.13 & {$[\mathrm{M}-\mathrm{H}]^{-}$} & 191.03415 & 191.0341 & 0.3 & $\mathrm{C}_{10} \mathrm{H}_{8} \mathrm{O}_{4}$ & - & $\begin{array}{l}\text { Coumarin base }+1 O \\
1 \mathrm{MeO}\end{array}$ & 3 & C & - & \\
\hline 74 & 10.22 & {$[\mathrm{M}-\mathrm{H}]^{-}$} & 359.07709 & 359.07724 & -0.4 & $\mathrm{C}_{18} \mathrm{H}_{16} \mathrm{O}_{8}$ & $72.9915,135.0437,161.0231,179.0334,197.0434$ & Rosmarinic acid & $1^{\mathrm{s}}$ & $\mathrm{HC}$ & 5281792 & 3 \\
\hline 75 & 10.23 & {$[\mathrm{M}-\mathrm{H}]^{-}$} & 313.0722 & 313.07122 & 3.1 & $\mathrm{C}_{17} \mathrm{H}_{14} \mathrm{O}_{6}$ & $\begin{array}{c}109.0279,123.0430,133.0286,151.0387 \\
161.0231\end{array}$ & $\begin{array}{l}\text { 3,4-Dihydroxycinnamoyl- } \\
\text { (Z)-2-(3,4-dihydroxyphenyl) } \\
\text { ethenol }\end{array}$ & 2 & $\mathrm{HC}$ & 14353342 & \\
\hline 76 & 10.61 & {$[\mathrm{M}-\mathrm{H}]^{-}$} & 431.09967 & 431.09836 & 3 & $\mathrm{C}_{21} \mathrm{H}_{20} \mathrm{O}_{10}$ & $152.9944,227.0337,255.0304,285.0388$ & Kaempferol-3-O-rhamnoside & 2 & $\mathrm{~F}$ & 5316673 & 2 \\
\hline \multirow[t]{2}{*}{77} & 10.71 & {$[\mathrm{M}-\mathrm{H}]^{-}$} & 193.04984 & 193.05063 & -4.1 & $\mathrm{C}_{10} \mathrm{H}_{10} \mathrm{O}_{4}$ & $\begin{array}{c}\text { 121.0280, 133.0775, 148.8996, 161.02284, } \\
177.0188\end{array}$ & \multirow[t]{2}{*}{ Ferulic acid } & \multirow[t]{2}{*}{2} & \multirow[t]{2}{*}{$\mathrm{HC}$} & \multirow[t]{2}{*}{445858} & \\
\hline & 10.75 & {$[\mathrm{M}+\mathrm{H}]^{+}$} & 195.06569 & 195.06519 & 2.6 & $\mathrm{C}_{10} \mathrm{H}_{10} \mathrm{O}_{4}$ & $\begin{array}{c}135.0446,145.0289,149.0605,163.0385, \\
177.0550 \\
\end{array}$ & & & & & \\
\hline \multirow{2}{*}{78} & 11.08 & {$[\mathrm{M}-\mathrm{H}]^{-}$} & 191.03412 & 191.03499 & -4.6 & $\mathrm{C}_{10} \mathrm{H}_{8} \mathrm{O}_{4}$ & $147.0439,149.0238,175.0114,191.0345$ & \multirow{2}{*}{ Scopoletin } & \multirow{2}{*}{2} & \multirow{2}{*}{ C } & \multirow{2}{*}{5280460} & \multirow{2}{*}{2} \\
\hline & 11.08 & {$[\mathrm{M}+\mathrm{H}]^{+}$} & 193.05025 & 193.04953 & 3.7 & $\mathrm{C}_{10} \mathrm{H}_{8} \mathrm{O}_{4}$ & - & & & & & \\
\hline 79 & 11.4 & {$[\mathrm{M}-\mathrm{H}]^{-}$} & 1251.60254 & 1251.60100 & 1.2 & $\mathrm{C}_{59} \mathrm{H}_{96} \mathrm{O}_{28}$ & $\begin{array}{c}589.4164,633.4128,733.4748,751.4918 \\
865.5422,883.5467927 .5394,957.5598 \\
1045.6177,1089.5382,1251.6044\end{array}$ & Triterpenoid saponin & 3 & TT & - & \\
\hline 80 & 11.63 & {$[\mathrm{M}-\mathrm{H}]^{-}$} & 1119.55419 & 1119.55874 & -4.1 & $\mathrm{C}_{54} \mathrm{H}_{88} \mathrm{O}_{24}$ & $\begin{array}{c}\text { 589.4158, 633.4137, } 733.4717,751.4915, \\
\text { 777.4700, 795.4831, } 913.5570,957.5609, \\
\text { 1119.5493 }\end{array}$ & $\begin{array}{l}\text { Triterpenoid saponin } \\
\quad \text { (Eryngioside } C \text { ) }\end{array}$ & 3 & TT & - & 5 \\
\hline 81 & 11.74 & {$[\mathrm{M}-\mathrm{H}]^{-}$} & 373.09302 & 373.09235 & 1.8 & $\mathrm{C}_{19} \mathrm{H}_{18} \mathrm{O}_{8}$ & $\begin{array}{l}\text { 123.0437, 135.0438, 149.0595, 160.0153, } \\
175.0390,179.0340,193.0498,197.0448\end{array}$ & Methyl rosmarinate & 3 & $\mathrm{HC}$ & 6479915 & \\
\hline 82 & 11.97 & {$[\mathrm{M}-\mathrm{H}]^{-}$} & 285.04071 & 285.04047 & 0.8 & $\mathrm{C}_{15} \mathrm{H}_{10} \mathrm{O}_{6}$ & $68.2225,164.8401,171.4909,175.0386,285.0380$ & Luteolin & 2 & $\mathrm{~F}$ & 5280445 & 6 \\
\hline \multirow{2}{*}{83} & 11.99 & {$[\mathrm{M}+\mathrm{H}]^{+}$} & 303.05045 & 303.04993 & 1.7 & $\mathrm{C}_{15} \mathrm{H}_{10} \mathrm{O}_{7}$ & $144.9594,160.2895,303.0504$ & \multirow{2}{*}{ Quercetin } & \multirow{2}{*}{$1^{\mathrm{s}}$} & \multirow{2}{*}{$\mathrm{F}$} & \multirow{2}{*}{5280343} & \multirow{2}{*}{1} \\
\hline & 11.99 & {$[\mathrm{M}-\mathrm{H}]^{-}$} & 301.03513 & 301.03537 & -0.8 & $\mathrm{C}_{15} \mathrm{H}_{10} \mathrm{O}_{7}$ & $\begin{array}{c}151.0019,178.9968,215.4516,243.6472 \\
301.0371\end{array}$ & & & & & \\
\hline 84 & 12.22 & {$[\mathrm{M}-\mathrm{H}]^{-}$} & 1103.52 & 1103.52744 & -6.7 & $\mathrm{C}_{53} \mathrm{H}_{84} \mathrm{O}_{24}$ & - & Triterpenoid saponin & 3 & TT & - & \\
\hline 85 & 13.63 & {$[\mathrm{M}-\mathrm{H}]^{-}$} & 285.04074 & 285.04047 & 0.9 & $\mathrm{C}_{15} \mathrm{H}_{10} \mathrm{O}_{6}$ & $176.8428,187.0409,285.0426$ & Kaempferol & $1^{\mathrm{s}}$ & $\mathrm{F}$ & 5280863 & 6 \\
\hline 86 & 13.84 & {$[\mathrm{M}-\mathrm{H}]^{-}$} & 209.11772 & 209.11832 & -2.9 & $\mathrm{C}_{12} \mathrm{H}_{18} \mathrm{O}_{3}$ & $59.0122,97.0641,165.1275,209.1182$ & Jasmonic acid & 2 & $\mathrm{O}$ & 5281166 & \\
\hline
\end{tabular}


Table 2. Cont.

\begin{tabular}{|c|c|c|c|c|c|c|c|c|c|c|c|c|}
\hline No & RT [min] & Ion mode & $\begin{array}{c}\text { Observed } \\
\mathrm{m} / \mathrm{z}\end{array}$ & $\begin{array}{l}\text { Reference } \\
\mathrm{m} / \mathrm{z}\end{array}$ & $\begin{array}{l}\text { Delta } \\
\text { (ppm) }\end{array}$ & $\begin{array}{l}\text { Molecular } \\
\text { Formula }\end{array}$ & Fragmentation & Name & MSI Level $^{\text {a }}$ & Class $^{b}$ & CID $^{c}$ & Ref. \\
\hline \multirow{2}{*}{87} & 14.05 & {$[\mathrm{M}-\mathrm{H}]^{-}$} & 161.02324 & 161.02387 & -3.9 & $\mathrm{C}_{9} \mathrm{H}_{6} \mathrm{O}_{3}$ & $133.0287,161.0232$ & \multirow{2}{*}{ Umbelliferone } & \multirow{2}{*}{2} & \multirow{2}{*}{ C } & \multirow{2}{*}{5281426} & \multirow{2}{*}{2} \\
\hline & 14.05 & {$[\mathrm{M}+\mathrm{H}]^{+}$} & 163.03908 & 163.03897 & 0.7 & $\mathrm{C}_{9} \mathrm{H}_{6} \mathrm{O}_{3}$ & $\begin{array}{c}119.0368,121.0656,137.3907,145.0288 \\
163.0385\end{array}$ & & & & & \\
\hline 88 & 14.87 & {$[\mathrm{M}-\mathrm{H}]^{-}$} & 1117.5357 & 1117.54309 & -6.6 & $\mathrm{C}_{54} \mathrm{H}_{86} \mathrm{O}_{24}$ & $\begin{array}{c}\text { 583.3667, 715.4624, 743.4582, 937.5284 } \\
\text { 985.5461, 1027.5735, 1075.5281 }\end{array}$ & Triterpenoid saponin & 3 & TT & - & \\
\hline 89 & 15.2 & {$[\mathrm{M}-\mathrm{H}]^{-}$} & 1203.53925 & 1203.54348 & -3.5 & $\mathrm{C}_{57} \mathrm{H}_{88} \mathrm{O}_{27}$ & $\begin{array}{c}\text { 645.4126, } 715.4597,743.4602,1099.5226 \\
1159.5333\end{array}$ & Triterpenoid saponin & 3 & TT & - & \\
\hline 90 & 15.27 & {$[\mathrm{M}-\mathrm{H}]^{-}$} & 1071.4957 & 1071.50122 & -5.2 & $\mathrm{C}_{52} \mathrm{H}_{80} \mathrm{O}_{23}$ & $436.7717,746.7781,772.1053$ & Triterpenoid saponin & 3 & TT & - & \\
\hline 91 & 15.31 & {$[\mathrm{M}-\mathrm{H}]^{-}$} & 1159.54309 & 1159.5384 & 4 & $\mathrm{C}_{52} \mathrm{H}_{88} \mathrm{O}_{28}$ & $436.1860,587.3589,715.4593,1099.5171$ & Triterpenoid saponin & 3 & TT & - & \\
\hline 92 & 17.3 & {$[\mathrm{M}-\mathrm{H}]^{-}$} & 1099.52958 & 1099.53252 & -2.7 & $\mathrm{C}_{54} \mathrm{H}_{84} \mathrm{O}_{23}$ & $734.0673,890.0820$ & $\begin{array}{l}\text { Triterpenoid saponin } \\
\text { (Eryngioside J) }\end{array}$ & 3 & TT & - & 5 \\
\hline 93 & 17.52 & {$[\mathrm{M}+\mathrm{H}]^{+}$} & 177.05464 & 177.05463 & 0.1 & $\mathrm{C}_{10} \mathrm{H}_{8} \mathrm{O}_{3}$ & $\begin{array}{c}93.0346,119.0863,121.0391,135.0801 \\
145.0289,177.0549\end{array}$ & 7-Methoxycoumarin & 2 & C & 5280567 & 2 \\
\hline 94 & 17.71 & {$[\mathrm{M}+\mathrm{H}]^{+}$} & 969.51023 & 969.50591 & 4.5 & $\mathrm{C}_{49} \mathrm{H}_{76} \mathrm{O}_{19}$ & - & $\begin{array}{c}\text { Triterpenoid saponin } \\
(3-O-\beta-\mathrm{D}-\text { glucopyranosyl-(1 } \\
\rightarrow 2)-\beta-\mathrm{D} \\
\text { glucuronopyranosyl- } \\
\text { 22-O-angeloyl-R1-barrigenol) }\end{array}$ & ) & TT & - & 7 \\
\hline 95 & 18.22 & {$[\mathrm{M}-\mathrm{H}]^{-}$} & 1187.54376 & 1187.54857 & -4 & $\mathrm{C}_{57} \mathrm{H}_{88} \mathrm{O}_{26}$ & - & Triterpenoid saponin & 3 & TT & - & \\
\hline 96 & 18.31 & {$[\mathrm{M}+\mathrm{H}]^{+}$} & 911.50453 & 911.50043 & 4.5 & $\mathrm{C}_{47} \mathrm{H}_{74} \mathrm{O}_{17}$ & - & $\begin{array}{c}\text { Triterpenoid saponin } \\
\text { (3-O- } \beta \text {-dglucopyranosyl-(1 } \\
\rightarrow \\
\text { 2)- } \beta \text {-D-glucuronopyranosyl- } \\
\text { 22-O-angeloyl-A1- } \\
\text { barrigenol) }\end{array}$ & 3 & TT & - & 7 \\
\hline 97 & 18.4 & {$[\mathrm{M}-\mathrm{H}]^{-}$} & 1041.58291 & 1041.57868 & 4.1 & $\mathrm{C}_{57} \mathrm{H}_{86} \mathrm{O}_{17}$ & $\begin{array}{c}489.3563,502.9471,583.3742,603.3950 \\
639.4351\end{array}$ & Triterpenoid saponin & 3 & TT & - & \\
\hline 98 & 21.07 & {$[\mathrm{M}+\mathrm{H}]^{+}$} & 293.2114 & 293.21112 & 1 & $\mathrm{C}_{18} \mathrm{H}_{28} \mathrm{O}_{3}$ & - & OPDA & 3 & $\mathrm{O}$ & 656750 & \\
\hline 99 & 23.01 & {$[\mathrm{M}-\mathrm{H}]^{-}$} & 471.34903 & 471.34799 & 2.2 & $\mathrm{C}_{30} \mathrm{H}_{48} \mathrm{O}_{4}$ & - & Triterpenoid sapogenin & 3 & TT & - & \\
\hline
\end{tabular}

a Metabolite identification level according to Metabolite Standards Initiative recommendation (1-identified metabolites, 2—putatively annotated compounds, 3-putatively characterized compound classes, 4 - unknown compounds); ${ }^{\mathrm{b}}$ Group of compounds: AA—amino acids, BA—benzoic acid derivates, C—coumarins, HC—hydroxycinnamic acid derivates, F-flavonoids, N—nucleotide, TT—triterpenoids, O—other compounds; ${ }^{\mathrm{C}} \mathrm{CID}-$ Compound ID, PubChem; ${ }^{\mathrm{s}}$ Compounds identified with using commercial standards. 


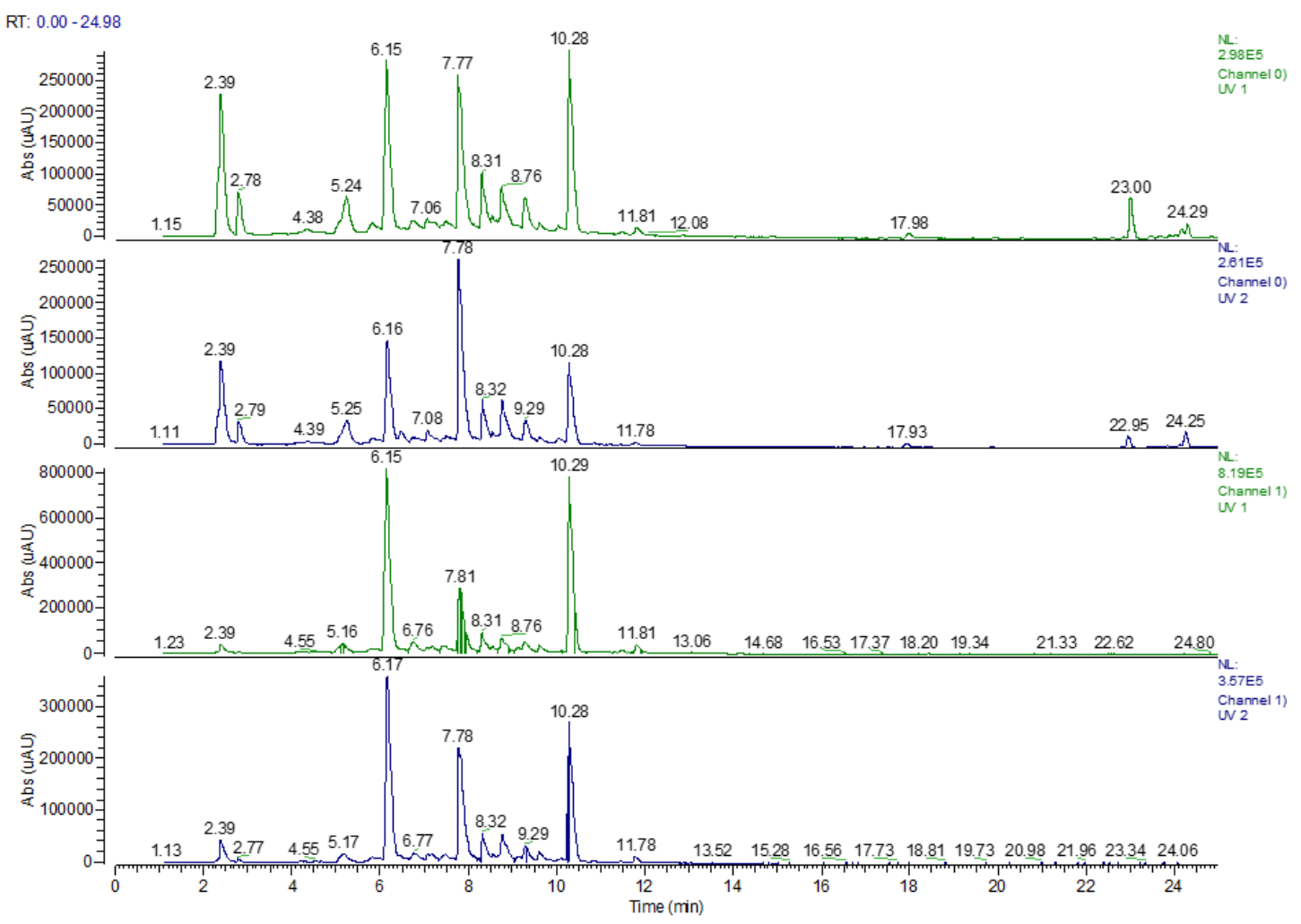

Figure 3. The UPLC-UV chromatograms of shoots from in vivo plants (green) and in vitro cultures (blue) of Eryngium alpinum L. extracts recorded at $270 \mathrm{~nm}(1,2)$ and $330 \mathrm{~nm}(3,4)$.

One of the major groups of compounds were hydroxycinnamic acid derivates, which include conjugates of coumaric, caffeic and ferulic acid with the hexose (neutral losses $-162.0834, \mathrm{C}_{6} \mathrm{H}_{10} \mathrm{O}_{5}$ ) and the quinic acid (characteristic fragment $m / z$ 191.0195, $\mathrm{C}_{7} \mathrm{H}_{11} \mathrm{O}_{6}{ }^{-}$). Three conjugates of caffeic acid and quinic acid were annotated in the sample namely neochlorogenic acid (5-caffeoylquinic acid, RT = $5.57 \mathrm{~min}$ ), chlorogenic acid (3-caffeoylquinic acid, $\mathrm{RT}=6.06$ ), and isochlorogenic acid ( $5 Z$-caffeoylquinic acid, $\mathrm{RT}=7.02 \mathrm{~min}$ ); they were previously described by Kikowska et al. [6,7]. The pseudo-molecular ion $\mathrm{m} / \mathrm{z} 353.08743$ corresponded with the formula $\mathrm{C}_{16} \mathrm{H}_{17} \mathrm{O}_{9}{ }^{-}$and gave the fragmentation pattern 191.0546, 179.0334, 161.0226, 135.0438 characteristic for caffeoylquinic acids. Compound 68 was detected in negative ion mode as a pseudo-molecular ion 515.11951, corresponded with the molecular formula $\mathrm{C}_{25} \mathrm{H}_{23} \mathrm{O}_{12}{ }^{-}$, and was annotated as dicaffeoylquinic acid. Compound $39(\mathrm{RT}=7.14 \mathrm{~min})$ was detected in negative ion mode as a coumaroylquinic acid $\left(\mathrm{m} / z\right.$ 337.09219, $\left.\mathrm{C}_{16} \mathrm{H}_{17} \mathrm{O}_{8}{ }^{-}\right)$. The ion was observed at three different retention times $(\mathrm{RT}=6.49,7.76$, and $8.43 \mathrm{~min})$ at $\mathrm{m} / \mathrm{z} 367.10380\left(\mathrm{C}_{17} \mathrm{H}_{19} \mathrm{O}_{9}{ }^{-}\right)$, which suggested the existence of three isomeric forms of feruloylquinic acid. The conjugates of choline were detected in positive ion mode and assigned as caffeoylcholine and coumaroylcholine. The most intense peak at RT $=10.22 \mathrm{~min}$ was exhibited by $[\mathrm{M}-\mathrm{H}]^{-}$at $\mathrm{m} / z 359.07709\left(\mathrm{C}_{18} \mathrm{H}_{15} \mathrm{O}_{8}{ }^{-}\right)$and by a complex ion $[2 \mathrm{M}-\mathrm{H}]^{-}$ at $m / z$ 719.15418. This compound was identified as rosmarinic acid by the exact mass, the fragmentation pattern and the comparison with the external standard (Sigma-Aldrich). Rosmarinic acid was previously described by Le Claire et al. [4] and Kikowska et al. [6,7]. In accordance with the literature, rosmarinic acid glucoside (521.13037, $\mathrm{C}_{24} \mathrm{H}_{25} \mathrm{O}_{13}{ }^{-}$) and glucuronide (535.10642, $\mathrm{C}_{24} \mathrm{H}_{23} \mathrm{O}_{14}{ }^{-}$) were found in negative ion mode [17]. Furthermore, 3,4-dihydroxycinnamoyl-(Z)-2-(3,4-dihydroxyphenyl)ethanol (313.0722, $\mathrm{C}_{17} \mathrm{H}_{13} \mathrm{O}_{6}{ }^{-}$) and methyl rosmarinate (373.09302, $\mathrm{C}_{19} \mathrm{H}_{18} \mathrm{O}_{8}{ }^{-}$) were recognized in the extracts. Coumarins such as umbeliferone, scopoletin, 7-methoxycoumarin, and dihydroxycoumarin were recognized in our sample basing on the exact mass and the fragmentation pattern and were previously described for the different Eryngium species and the Apiaceae family [18], however, for the first time they were recognized in E. alpinum. Moreover, the conjugates with glucose were tentatively identified 
in the samples as esculin, scopoletin-7-O-dihexoside, and scopolin. The pseudo-molecular ions were observed at two different retention times at $m / z 223.0601\left(\mathrm{C}_{11} \mathrm{H}_{11} \mathrm{O}_{5}{ }^{+}\right)$and were tentatively assigned as isofraxidin and fraxidin.

The representative flavonoids were mostly recognized as quercetin, kaempferol, and luteolin derivates in positive and negative ion mode. The MS/MS spectra showed the typical fragmentation pattern for O-flavonoids with hexose $\left(-162.0539, \mathrm{C}_{6} \mathrm{H}_{10} \mathrm{O}_{5}\right)$, rhamnose $\left(-146.0656, \mathrm{C}_{6} \mathrm{H}_{11} \mathrm{O}_{4}\right)$, rutinose (-308.1105, $\left.\mathrm{C}_{12} \mathrm{H}_{20} \mathrm{O}_{9}\right)$, or dihexose (-324.1061, $\left.\mathrm{C}_{12} \mathrm{H}_{20} \mathrm{O}_{10}\right)$ losses in negative ion mode. Consequently, quercetin-3-O-rutinose, quercetin-3-O-galactoside, quercetin-3-O-glucoside, quercetin, and kaempferol were verified by means of the reference standards (Sigma-Aldrich). Other flavonoids were tentatively assigned as kaempferol-O-rhamnodihexoside, kaempferol-3-O-dihexoside, kaempferol-3-O-rutinoside, kaempferol-O-hexoside (I and II), kaempferol-3-O-rhamnoside, luteolin-7-O-rhamnohexoside, luteolin-7-O-hexoside, quercetin-3-O-dihexoside-7-O-rhamnoside, quercetin-3-O-glucoside-7-O-rhamnoside, and quercetin-3-O-dihexoside and verified with the literature data for the described Eryngium species $[19,20]$. Moreover, luteolin-C-hexoside, apigenin-7-O-rhamnohexoside, and quercetin 3-(6-O-acetyl)-hexoside were found in the extracts. Peak 59 showed a precursor ion at $\mathrm{m} / z 447.09238\left(\mathrm{C}_{21} \mathrm{H}_{19} \mathrm{O}_{11}{ }^{-}\right)$and was tentatively annotated as a luteolin-C-hexoside according to the MS/MS analysis, which corresponded to the loss of fragments-90 and -120 , characterizing the break of $C$-hexoside. Compound 65 was assigned as quercetin 3-(6-O-acetyl)-hexoside (505.09988, $\left.\mathrm{C}_{23} \mathrm{H}_{21} \mathrm{O}_{12}{ }^{-}\right)$whereas compound 69 was characterized as an apigenin-7-O-rhamnohexoside $\left(577.16005, \mathrm{C}_{27} \mathrm{H}_{29} \mathrm{O}_{20}{ }^{-}\right)$. The product ion spectra demonstrated a fragment ion at $m / z 269.0468$ corresponding to apigenin aglycone. Five compounds $(46,56,57,63$, 64), which corresponded with ions at $m / z$ : $753.18783\left(\mathrm{C}_{33} \mathrm{H}_{37} \mathrm{O}_{20}{ }^{-}\right), 609.14679\left(\mathrm{C}_{27} \mathrm{H}_{29} \mathrm{O}_{16}{ }^{+}\right), 753.21992$ $\left(\mathrm{C}_{33} \mathrm{H}_{37} \mathrm{O}_{20}{ }^{-}\right)$, $607.13054\left(\mathrm{C}_{27} \mathrm{H}_{28} \mathrm{O}_{16}{ }^{-}\right)$, and $739.21136\left(\mathrm{C}_{33} \mathrm{H}_{39} \mathrm{O}_{19}{ }^{+}\right)$, were recognized as unknown flavonoids. The MS/MS spectrum of compounds 55, 65, 66 and 72 showed a major fragment at $m / z 301.03711\left(\mathrm{C}_{15} \mathrm{H}_{9} \mathrm{O}_{7}{ }^{-}\right)$in negative ion mode, which could be quercetin aglycone. Furthermore, a fragment at $m / z 285.03955\left(\mathrm{C}_{15} \mathrm{H}_{9} \mathrm{O}_{6}{ }^{-}\right)$was found in the MS/MS spectrum of compound 64 and corresponded with the tetrahydroxyflavone moiety.

Several triterpenoid saponins were found in the samples. Similar to Ożarowski et al. [17], we observed precursors and the fragmentation pattern as in the related species, E. planum. The pseudo-molecular ion at $m / z 471.34903$ corresponded with the molecular formula $\mathrm{C}_{30} \mathrm{H}_{47} \mathrm{O}_{4}{ }^{-}$and was recognized as a major sapogenin in the extract. Twelve precursors were tentatively assigned as triterpenoid saponins. The pseudo-molecular ions $1119.55419\left(\mathrm{C}_{54} \mathrm{H}_{87} \mathrm{O}_{24}{ }^{-}\right)$and $1099.52958\left(\mathrm{C}_{54} \mathrm{H}_{83} \mathrm{O}_{23}{ }^{-}\right)$could be annotated as eryngioside $\mathrm{C}$ and eryngioside $\mathrm{J}$ according to the exact mass and fragmentation [21]. Moreover, ions $969.51023\left(\mathrm{C}_{49} \mathrm{H}_{77} \mathrm{O}_{19}{ }^{+}\right)$and $911.50453\left(\mathrm{C}_{47} \mathrm{H}_{75} \mathrm{O}_{17}{ }^{+}\right)$could be putatively assigned as 3-O- $\beta$-D-glucopyranosyl-( $\rightarrow 2)-\beta$-D-glucuronopyranosyl-22-O-angeloyl-R1-barrigenol and 3-O- $\beta$-D-glucopyranosyl-( $\rightarrow 2$ - $\beta$-D-glucuronopyranosyl-22-O-angeloyl-A1-barrigenol - saponins observed in E. planum and E. maritimum [22]. This is the first report on triterpenoid saponins detection in E. alpinum. However, further works are required for identification of the saponin structures in this species.

In line with the literature, hydroxybenzoates were found in the extracts of Eryngium [20]. Glucosyringic acid (359.09950, $\mathrm{C}_{15} \mathrm{H}_{19} \mathrm{O}_{10}{ }^{-}$), trimethoxybenzoic acid $\left(213.07613, \mathrm{C}_{10} \mathrm{H}_{13} \mathrm{O}_{5}{ }^{+}\right.$), dihydroxybenzoic acid hexoside (315.0725, $\left.\mathrm{C}_{13} \mathrm{H}_{15} \mathrm{O}_{9}{ }^{-}\right)$, vanillic acid hexoside (329.08841, $\left.\mathrm{C}_{14} \mathrm{H}_{17} \mathrm{O}_{9}^{-}\right)$, vanillic acid $\left(167.0350, \mathrm{C}_{8} \mathrm{H}_{7} \mathrm{O}_{4}{ }^{-}\right)$, hydroxyphenyllacetic acid $\left(181.05003, \mathrm{C}_{9} \mathrm{H}_{9} \mathrm{O}_{4}{ }^{-}\right)$, dimethoxybenzaldehyde (167.07097, $\mathrm{C}_{9} \mathrm{H}_{11} \mathrm{O}_{3}{ }^{+}$), and glucovanillin (313.09305, $\mathrm{C}_{14} \mathrm{H}_{17} \mathrm{O}_{8}{ }^{-}$) were tentatively recognized in the sample. Also, citric acid was identified as a major carboxylic acid. Twelve amino acids and dipeptides were observed in positive and negative ion mode. Three nucleotides were recognized as uridine, adenosine and guanosine. The pseudo-molecular ions at $m / z 182.04490$ $\left(\mathrm{C}_{8} \mathrm{H}_{8} \mathrm{NO}_{4}{ }^{-}\right)$and $218.10310\left(\mathrm{C}_{9} \mathrm{H}_{16} \mathrm{NO}_{5}{ }^{-}\right)$were annotated as 4-pyridoxic acid and pantothenic acid, the major vitamins in the extracts. Some of phytohormones such as 5-hydroxy-3-indoleacetic acid, gibberellic acid, 12-hydroxyjasmonic acid, jasmonic acid, and OPDA were putatively identified by means of the exact mass and the fragmentation pattern. 
The results of the study indicated that the extracts obtained from E. alpinum shoots, both from in vitro cultures in vivo plantlets, inhibited growth of Acanthamoeba sp. trophozoites to varying degrees (Tables 3-5; Figures 4 and 5).

Table 3. The effect of the extract from shoots from in vivo plant of Eryngium alpinum L. [0.5 mg/mL, 2.5 $\mathrm{mg} / \mathrm{mL}, 5 \mathrm{mg} / \mathrm{mL}$ ] on inhibition of Acanthamoeba trophozoites during four days of treatment.

\begin{tabular}{cccc}
\hline \multirow{2}{*}{ Extract Concentration $[\mathrm{mg} / \mathrm{mL}]$} & \multicolumn{3}{c}{ Duration of Treatment [Days] } \\
\cline { 2 - 4 } & 2nd Day & 3rd Day & 4th Day \\
\cline { 2 - 4 } & $\mathbf{M N} \pm \mathbf{S D}$ & $\mathbf{M N} \pm \mathbf{S D}$ & $\mathbf{M N} \pm \mathbf{S D}$ \\
\hline control & $5.62 \pm 1.96$ & $11.93 \pm 2.33$ & $17.06 \pm 2.95$ \\
0.5 & $5.00 \pm 2.26$ & $11.83 \pm 2.73$ & $17.06 \pm 2.50$ \\
2.5 & $4.62 \pm 1.50$ & $11.54 \pm 3.66$ & $16.44 \pm 6.61$ \\
5 & $2.67 \pm 1.88^{*}$ & $5.33 \pm 3.98^{*}$ & $6.69 \pm 3.08^{*}$ \\
\hline
\end{tabular}

$\mathrm{MN}-$ mean number of trophozoites. ${ }^{*} p<0.05$ statistically significant difference in comparison with the control during the same time interval; $n=18$.

Table 4. The effect of the extract from in vitro shoot cultures of Eryngium alpinum L. [0.5 mg/mL, 2.5 $\mathrm{mg} / \mathrm{mL}, 5 \mathrm{mg} / \mathrm{mL}$ ] on inhibition of Acanthamoeba trophozoites during four days of treatment.

\begin{tabular}{cccc}
\hline \multirow{2}{*}{$\begin{array}{c}\text { Extract Concentration } \\
{[\mathrm{mg} / \mathrm{mL}]}\end{array}$} & \multicolumn{3}{c}{ Duration of Treatment [Days] } \\
\cline { 2 - 4 } & 2nd Day & 3rd Day & 4th Day \\
\cline { 2 - 4 } & $\mathbf{M N} \pm \mathbf{S D}$ & $\mathbf{M N} \pm \mathbf{S D}$ & $\mathbf{M N} \pm \mathbf{S D}$ \\
\hline control & $5.62 \pm 1.96$ & $11.53 \pm 2.33$ & $17.06 \pm 2.95$ \\
0.5 & $2.53 \pm 1.88^{*}$ & $7.46 \pm 3.00^{*}$ & $13.45 \pm 3.58^{*}$ \\
2.5 & $1.89 \pm 1.37 *$ & $6.89 \pm 2.33^{*}$ & $12.69 \pm 3.46^{*}$ \\
5 & $1.06 \pm 1.35^{*}$ & $3.76 \pm 2.31 *$ & $5.57 \pm 2.02 *$ \\
\hline
\end{tabular}

MN-mean number of trophozoites. ${ }^{*} p<0.05$ statistically significant difference in comparison with the control during the same time interval; $n=18$.

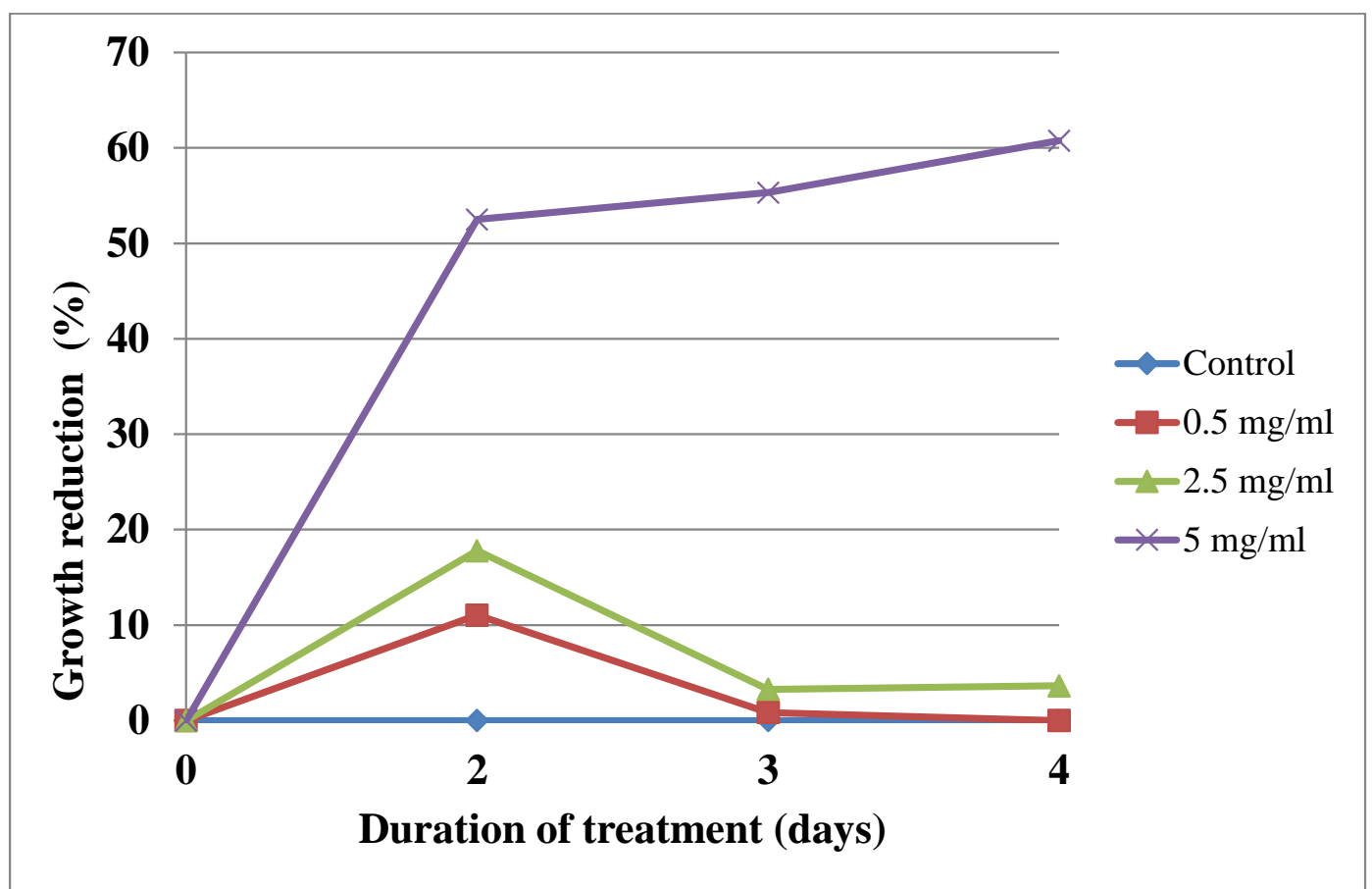

Figure 4. The effect of the extract from shoots of in vivo plant Eryngium alpinum L. [0.5 mg/mL, 2.5 $\mathrm{mg} / \mathrm{mL}, 5 \mathrm{mg} / \mathrm{mL}$ ] on inhibition of Acanthamoeba trophozoites proliferation in the culture medium. 
Table 5. Determination of $\mathrm{IC}_{50}[\mathrm{mg} / \mathrm{mL}]$ for the studied extracts of Eryngium alpinum $\mathrm{L}$.

\begin{tabular}{cccc}
\hline Plant Material & IC $_{\mathbf{5 0}}$ 2nd Day & IC $_{\mathbf{5 0}}$ 3rd Day & IC $_{\mathbf{5 0}}$ 4th Day \\
\hline Shoots from in vivo plants & $4.80 \mathrm{mg} / \mathrm{ml}$ & $4.80 \mathrm{mg} / \mathrm{ml}$ & $4.60 \mathrm{mg} / \mathrm{ml}$ \\
Shoots from in vitro cultures & $0.35 \mathrm{mg} / \mathrm{ml}$ & $3.50 \mathrm{mg} / \mathrm{ml}$ & $4.15 \mathrm{mg} / \mathrm{ml}$ \\
\hline
\end{tabular}

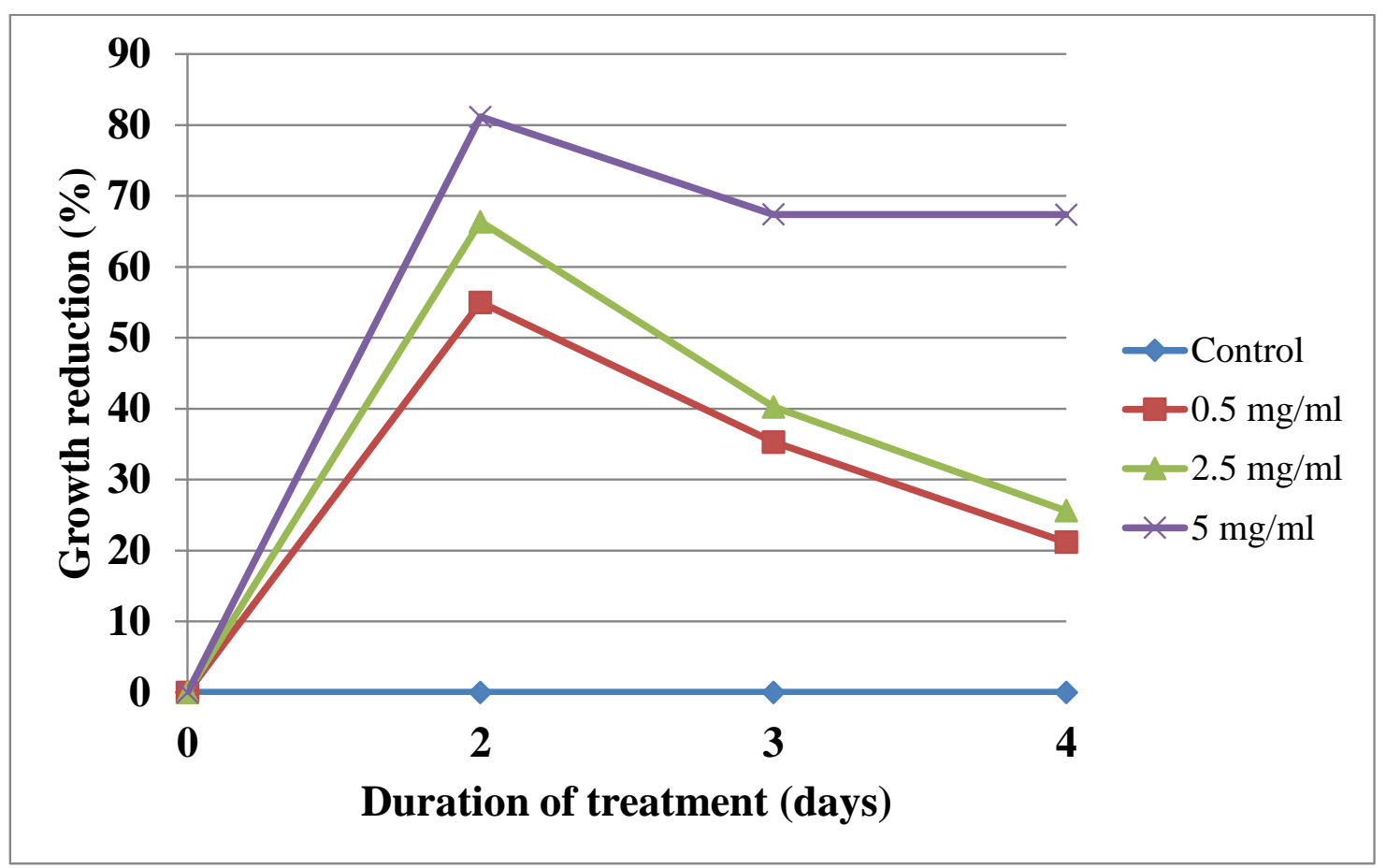

Figure 5. The effect of the extract from in vitro shoot cultures of Eryngium alpinum L. [0.5 mg/mL, 2.5 $\mathrm{mg} / \mathrm{mL}, 5 \mathrm{mg} / \mathrm{mL}$ ] on inhibition of Acanthamoeba trophozoites proliferation in the culture medium.

The dependence of the effect on the extract concentration and treatment time was noted. The strongest effect was observed for leaves from in vitro shoot culture. The extract showed the highest antiamoebicidal effect already on the second day of treatment: indicated inhibition of trophozoites was $81.14 \%, 66.38 \%$, and $54.99 \%$ at the concentrations of $5 \mathrm{mg} / \mathrm{mL}, 2.5 \mathrm{mg} / \mathrm{mL}$, and $0.5 \mathrm{mg} / \mathrm{mL}$, respectively (Table 4, Figure 4). The extract from shoots of in vivo plants at a dose of 0.5 and $2.5 \mathrm{mg} / \mathrm{mL}$ weakly inhibited the development of trophozoites (Table 3, Figure 3). The best $\mathrm{IC}_{50}$ index was calculated for leaves from the shoot culture extract. On the second day of treatment, the $\mathrm{IC}_{50}$ value was $0.35 \mathrm{mg} / \mathrm{mL}$ (Table 5).

Due to the problems in the treatment of opportunistic Acanthamoeba spp. and the lack of effective but safe drugs, the search continues for substances of plant origin that, applied as combined therapy, could contribute to decreasing the effective doses of antibiotics used [10,12].

In the literature on the subject, more scientific information on the plant extracts with the amoebicidal or amoebistatic activity against pathogenic strains of Acanthamoeba spp. can be found regarding the extracts from leaves of Origanum spp., Salvia spp., Melia azedarach, Ricinus communis, Pastinaca armenea, Inula oculuscristi; aerial parts of Croton spp., Pterocaulon polystachyum, flowers, roots and leaves of Rubus chamaemourus, Pueraria lobata, Solidago spp., flowers, roots, leaves and bark of Ipomoea sp., Kaempferia galanga, Cananga odorata, leaves and calluses of Passiflora spp., leaves and roots of Eryngium planum $[9,11,23-27]$.

It was shown in our studies that the extract of leaves from in vitro shoot culture of E. alpinum at a dose of $0.5 \mathrm{mg} / \mathrm{mL}$ was effective in inhibiting trophozoites, which can be interpreted as favourable compared to the amoebicidal effect of the plant extracts such as Allium sativum at $3.9 \mathrm{mg} / \mathrm{mL}$ [28], Salvia staminea at $16 \mathrm{mg} / \mathrm{mL}$ [29], Peucedanum caucasicum, P. palimbioides, P. chryseum, P. longibracteolatum [30], 
Origanum syriacum, O. laevigatum [31], Buddleia cordata at $32 \mathrm{mg} / \mathrm{mL}$ [32], and Trigonella foenum-graecum at $400 \mathrm{mg} / \mathrm{mL}$ [33].

The flavonoid-saponin fraction of the ethanolic extract from leaves of Eryngium planum L., at the concentration of $1 \mathrm{mg} / \mathrm{mL}$, with the similar phytochemical pattern to E. alpinum, showed the amoebistatic effect $-76 \%$ inhibition of amoebae growth on the third day of treatment. However, the flavonoid fraction from leaves at the concentration of $5 \mathrm{mg} / \mathrm{mL}$ revealed the $56.1 \%$ inhibitory effect and the phenolic acid fraction at the concentration of $2 \mathrm{mg} / \mathrm{mL}$ showed $36.8 \%$ inhibition. The authors concluded that the activity may be correlated with the saponin actions, which may be associated with the integration between those compounds and the cell wall of Acanthamoeba [27]. As stated by Mahboob et al. [34], the acanthamoebicidal effect of Lonicera japonica flower, which evoked a significant reduction of trophozoite viability, depends mostly on the major compound form the extract, that is chlorogenic acid. According to Bittner Fialová, rosmarinic acid and its derivates appeared to be promising anti-Acanthamoeba agents with the $\mathrm{EC}_{50}$ values between $5.6 \pm 0.3 \mathrm{mM}$ and $19.1 \pm 0.4 \mathrm{mM}$ [35]. The biological study of the Passiflora spp. extracts from leaves and callus biomass revealed that all the samples showed amoebostatic and amoebicidal properties at the concentrations from 4 to $12 \mathrm{mg} / \mathrm{mL}$. The authors tried to find a correlation between the studied activity and the presence of phenolic compounds, with particular emphasis on flavonoids [26]. Moreover, it is noteworthy that quercetin exhibited potent antiamoebic activities against Acanthamoeba [36]. These findings were accordingly similar to the results of the study performed on fractions of the ethanol extracts prepared from Frankenia thymifolia. The fractions showed moderate activity against Acanthamoeba castellanii, which may be associated with the presence of quercetin and its derivatives [37]. As it was shown in our study, $E$. alpinum shoots, in addition to the presence of phenolic acids and flavonoids, are characterized by a broad spectrum of coumarins. And as it results from numerous studies, phenolic compounds in the extracts of the species such as Allium sativum, Solidago virgaurea, Teucrium chamaedrys or Peucedanum spp. are responsible for the amoebicidal effect [11].

To our best knowledge, the present report is the first one that discusses the phytochemical screening and discusses the antiamoebic activity of Eryngium alpinum L. shoots from in vitro cultures and in vivo plants of this endangered species.

\section{Materials and Methods}

\subsection{The Plant Material Origin}

The fragments of the cuttings of Eryngium alpinum L. obtained from the Botanical Garden of Adam Mickiewicz University in Poznań in 2017 (52 25 $\left.13.1^{\prime \prime} \mathrm{N} 16^{\circ} 52^{\prime} 44.9^{\prime \prime} \mathrm{E}\right)$ were used for the initiation of in vitro cultures. The voucher specimens were deposited at the Department of Pharmaceutical Botany and Plant Biotechnology of PUMS under the following number: H-AP-2017-102.

\subsection{Establishment of In Vitro Cultures}

Young shoots with lateral buds were harvested. The collected explants were disinfected and transferred into basal MS medium [38] with plant growth regulators (PGRs), namely cytokinin BAP (6-benzylaminopurine), auxin IAA (indole-3-acetic acid), and gibberellin $\mathrm{GA}_{3}$ (gibberellic acid) at the concentration of $1.0 \mathrm{mg} / \mathrm{l}$ (Table 1), $0.76 \%$ agar and $\mathrm{pH}$ set to 5.8 before autoclaving at $121^{\circ} \mathrm{C}, 105 \mathrm{kPa}$ for $20 \mathrm{~min}$. All PGRs and agar originated from Sigma-Aldrich (Saint Louis, MO, USA). The cultures were placed in a growth chamber under controlled conditions, i.e., $21^{\circ} \mathrm{C}$ with a $16 \mathrm{~h} \mathrm{light} / 8 \mathrm{~h}$ dark photoperiod, $55 \mu \mathrm{mol} / \mathrm{m}^{2} \mathrm{~s}$ light, and subcultured every five weeks. Multiplication of shoots was repeated three times for each hormonal treatment using at least 10 explants (2-3 per flask).

\subsection{Detection of Metabolites in the Extracts Using UPLC-HESI-II-HRMS}

In order to conduct the phytochemical analysis, the exact amounts of fresh biomass from basal leaves of the intact plants as well as shoots from the in vitro cultures were dried at $40^{\circ} \mathrm{C}$ for $24 \mathrm{~h}$ to a 
constant weight. The dried samples were extracted with 70\% (v/v) EtOH (25 mg DW to $2.0 \mathrm{~mL})$ in safe-lock tubes (Eppendorf, Hamburg, Germany). The samples were shaken at $3000 \mathrm{rpm}$ for $20 \mathrm{~min}$ (IKA MS 3 Basic Vortex Mixer, Staufen, Germany) and centrifuged at 12,000 rpm, at $4^{\circ} \mathrm{C}$ for $15 \mathrm{~min}$ (Allegra 21 centrifuge, Beckman Coulter, Brea, CA, USA). Supernatants were filtered through a 0.22 $\mu \mathrm{m}$ PTFE syringe filter ( $\Phi 13 \mathrm{~mm}$, Kinesis Ltd, St. Neots, U.K.). Aquity UPLC (Waters, Milford, MA, USA) with a high resolution Orbitrap mass spectrometer (Thermo Fischer, Bremen, Germany) were applied to the phytochemical analysis of the ethanolic extracts. BEH C13 column $(1.7 \mu \mathrm{m}, 2.1 \times 150 \mathrm{~mm}$, Waters) was used for separation of the samples $(3 \mu \mathrm{L}$, partial loop mode $)$ at $45^{\circ} \mathrm{C}$ column temperature and $300 \mu \mathrm{L} / \mathrm{min}$ flow rate. $0.1 \%$ of formic acid in water (solvent A, MiliQ system, Merck, Darmstadt, Germany) and acetonitrile (solvent B, LC/MS grade, Merck) were used in gradient: initial-5\% B, 20 $\min -75 \% \mathrm{~B}, 22 \mathrm{~min}-98 \% \mathrm{~B}$, and isocratic $98 \% \mathrm{~B}$ for $24 \mathrm{~min}$. The PDA detector scanned in the range 220-400 nm at frequency 20 spectra/s.

The Orbitrap mass spectrometer equipped with the heated electrospray ion source II (HESI-II) operated in negative and positive ion mode. HESI II settings were: capillary voltage $-2.5 \mathrm{kV}$ (negative) and $3.5 \mathrm{kV}$ (positive), sheath gas flow-35, auxiliary gas flow-10, sweep gas flow-3 arbitrary units, ion transfer tube temperature $-400{ }^{\circ} \mathrm{C}$, auxiliary gas heater temperature $-350{ }^{\circ} \mathrm{C}$, and S-lens RF level 50. The full-MS spectra were recorded at mass resolution of 70,000 in the range 150-2000 $\mathrm{m} / \mathrm{z}$ and 200 ms maximum inject time. The data dependent MS2 spectra were recorded at resolution of 17,500. The data files were processed using Xcalibur Qual Browser (Thermo Fischer) and MSDIAL 3.9 software [39].

\subsection{Acanthamoebic Activity Examination}

In order to conduct the biological analysis, the exact amounts of fresh biomass from basal leaves of the intact plants as well as shoots from the in vitro cultures were dried at $40{ }^{\circ} \mathrm{C}$ for $24 \mathrm{~h}$ to a constant weight. Dried shoots from in vivo plants and in vitro cultures were extracted three times with $\mathrm{EtOH}$ $70 \%(v / v)$ at $95^{\circ} \mathrm{C}$. The extracts were concentrated under reduced pressure and used for the evaluation of the antiamoebic studies. The extract samples were weighed and then dissolved in $40 \mathrm{~mL}$ of DMSO (dimethyl sulfoxide). Distilled water was added to the solution to obtain the appropriate concentration. Then $200 \mathrm{~mL}$ of the appropriately diluted solution was added to $2 \mathrm{~mL}$ of trophozoites cultures to obtain the expected final concentrations $(0.5,2.5$ and $5 \mathrm{mg} / \mathrm{mL})$. In this study the strain of Acanthamoeba, isolated from the environmental sample, was used. This Acanthamoeba sp. strain was deposited in GenBank (NCBI) under the accession number KY203908. The pathogenicity of this strain was tested on laboratory animals. The research showed that this strain of amoebae is pathogenic for mice. The amoebae were axenically cultured on the liquid medium containing $2 \%$ Bacto-Casitone. Parasitological examination of the extracts was performed according to Derda et al. [9]. The study investigated the activity of the ethanol extracts from in vitro shoot cultures and in vivo plants. The increase in the number of parasites in culture was studied. Thoma hemocytometry chamber was used for cell counting. The amoebae were counted three times at $24 \mathrm{~h}$ intervals. The control consisted of cultured trophozoites without any extracts. The relationship between the extract concentration and the time of treatment for amoebae cultures was investigated.

\subsection{The Statistical Analysis}

The mean number of E. alpinum shoots and their length as well as the standard error were calculated in each hormonal variant of the culture medium. The data from biotechnological experiments were analyzed using a one-way analysis of variance (ANOVA) and the statistical significance was determined using Duncan's POST-HOC test ( $p$-value < 0.05). All the analyses were conducted employing STATISTICA v. 13 (StatSoft, Inc. 2015). The mean number of amoebae and standard deviation were calculated in each measurement group. The statistical analysis was determined employing the Mann-Whitney and ANOVA tests. Statistical significance was defined as $p<0.05$. 


\section{Conclusions}

In vitro shoot culture of Eryngium alpinum L. can be considered a valuable alternative source of biomass that is rich in desired secondary metabolites such as phenolic acids, coumarins, flavonoids, and triterpenoid saponins. This is especially important for protected species, the collection of which from the natural environment is impossible. The results suggest that the extracts from E. alpinum may be promising natural products for Acanthamoeba treatment. Further studies are necessary to clarify which bioactive compounds are responsible for the observed activity.

Author Contributions: Conceptualization, M.K.; methodology, M.K.; D.K. and M.D.; software, M.K.; D.K. and M.D.; formal analysis, M.K.; D.K. and M.D.; investigation, M.K.; D.K. and M.D.; data curation, M.K.; D.K. and M.D.; writing—original draft preparation, M.K. and D.K.; writing—review and editing, M.K.; B.T.; D.K., E.H. and M.D.; visualization, M.K.; D.K. and M.D.; supervision, E.H. and B.T.; project administration, M.K.; funding acquisition, M.K. All authors have read and agreed to the published version of the manuscript.

Funding: This research was funded by the National Science Centre, Poland, the grant number 2019/03/X/NZ7/01846.

Conflicts of Interest: The authors declare no conflict of interest.

\section{References}

1. Cullen, J.; Alexander, J.C.M.; Brady, A.; Brickell, C.D.; Green, P.S.; Heywood, V.H.; Jörgensen, P.M.; Jury, S.L.; Knees, S.G.; Leslie, A.C.; et al. The European Garden Flora; Cambridge University Press: London, UK, 1997; Volume V, pp. 396-398.

2. Andrello, M.; Devaux, C.; Quetier, F.; Till-Bottraud, I. Paying for conservation: A bioeconomic analysis of the effects of land management options on the viability of an endangered species, Eryngium alpinum. J. Appl. Ecol. 2017, 55, 1940-1950. [CrossRef]

3. Crowden, R.K.; Harborne, J.B.; Heywood, V.H. Chemosystematics of the Umbelliferae-A general survey. Phytochemistry 1969, 8, 1963-1984. [CrossRef]

4. Le Claire, E.; Schwaiger, S.; Banaigs, B.; Stuppner, H.; Gafner, F. Distribution of a new rosmarinic acid derivative Eryngium alpinum L. and other Apiaceae. J. Agric. Food Chem. 2005, 53, 4367-4372. [CrossRef] [PubMed]

5. Dunkic, V.; Vuko, E.; Bezic, N.; Kremer, D.; Ruscic, M. Composition an antiviral activity of the essential oils of Eryngium alpinum and E. amethystinum. Chem. Biodivers. 2013, 10, 1894-1902. [CrossRef]

6. Kikowska, M.; Thiem, B.; Szopa, A.; Klimek-Szczykułowicz, M.; Rewers, M.; Sliwinska, E.; Ekiert, H. Comparative analysis of phenolic acids and flavonoids in shoot cultures of Eryngium alpinum L.-An endangered and protected species with medicinal value. Plant Cell Tiss. Organ Cult. 2019, 139, 167-175. [CrossRef]

7. Kikowska, M.; Thiem, B.; Szopa, A.; Ekiert, H. Accumulation of valuable secondary metabolites: Phenolic acids and flavonoids in different in vitro systems of shoot cultures of the endangered plant species-Eryngium alpinum L. Plant Cell Tiss. Organ Cult. 2020, (in press). [CrossRef]

8. Verpoorte, R.; Contin, A.; Memelnik, J. Biotechnology for the production of plant secondary metabolites. Phytochem. Rev. 2002, 1, 13-25. [CrossRef]

9. Derda, M.; Hadaś, E.; Thiem, B. Plant extracts as natural amoebicidal agents. Parasitol. Res. 2009, 104, 705-708. [CrossRef]

10. Niyyati, M.; Dodangeh, S.; Lorenzo-Morales, J. A review of the current research trends in the application of medicinal plants as a source for novel therapeutic agents against Acanthamoeba infections. Iran. J. Pharm. Res. 2016, 15, 893-900.

11. Wei, C.E.; Yeng, C.Y.; Mahboob, T.; Ling, L.C.; Raju, C.S.; Barusrux, S.; Nissapatron, V. Natural products: Alternative therapeutic compounds against Acanthamoeba spp. Asian J. Pharmacogn. 2019, 3, 29-38. [CrossRef]

12. Derda, M.; Hadaś, E. The use of phytotherapy in diseases caused by parasitic protozoa. Acta Parasitol. 2015, 60,1-8. [CrossRef] [PubMed]

13. Padzik, M.; Hendiger, E.B.; Szaflik, J.P.; Chomicz, L. Amoebae of the genus Acanthamoeba-Pathological agents in humans. Post. Mikrobiol. 2017, 56, 429-439.

14. Thiem, B.; Kikowska, M.; Krawczyk, A.; Więckowska, B.; Sliwinska, E. Phenolic acid and DNA contents of micropropagated Eryngium planum L. Plant Cell Tiss. Organ Cult. 2013, 114, 197-206. [CrossRef] 
15. Kikowska, M.; Thiem, B.; Sliwinska, E.; Rewers, M.; Kowalczyk, M.; Stochmal, A.; Długaszewska, J. Micropropagation of Eryngium campestre L. via shoot culture provides valuable uniform plant material with enhanced content of phenolic acids and antimicrobial activity. Acta Biol. Cracov. Bot. 2016, 58, 43-56. [CrossRef]

16. Kikowska, M.; Thiem, B.; Sliwinska, E.; Rewers, M.; Kowalczyk, M.; Stochmal, A.; Oleszek, W. The effect of nutritional factors and plant growth regulators on micropropagation and production of phenolic acids and saponins from plantlets and adventitious root cultures of Eryngium maritimum L. J. Plant Growth Regul. 2014, 33, 809-819. [CrossRef]

17. Ożarowski, M.; Thiem, B.; Mikołajczak, P.L.; Piasecka, A.; Kachlicki, P.; Szulc, M.; Kaminska, E.; Bogacz, A.; Kujawski, R.; Bartkowiak-Wieczorek, J.; et al. Improvement in long-term memory following chronic administration of Eryngium planum root extract in scopolamine model: Behavioral and molecular study. Evid.-Based Complement. Alternat. Med. 2015, 2015, 145140. [CrossRef]

18. Wang, P.; Su, Z.; Yuan, W.; Deng, G.; Li, S. Phytochemical constituents and pharmacological activities of Eryngium L. (Apiaceae). Pharm. Crops 2012, 3, 99-120. [CrossRef]

19. Erdem, S.A.; Nabavi, S.F.; Orhan, I.E.; Daglia, M.; Izadi, M.; Nabavi, S.M. Blessings in disguise: A review of phytochemical composition and antimicrobial activity of plants belonging to the genus Eryngium. DARU 2015, 23, 1-22. [CrossRef]

20. Cádiz-Gurrea, M.; Fernandez-Arroyo, S.; Joven, J.; Segura-Carretero, A. Comprehensive characterization by UHPLC-ESI-Q-TOF-MS from an Eryngium bourgatii extract and their antioxidant and anti-inflammatory activities. Food Res. Intern. 2013, 50, 197-204. [CrossRef]

21. Zhang, Z.; Li, S.; Ownby, S.; Wang, P.; Yuan, W.; Zhang, W.; Beasley, R.S. Phenolic compounds and rare polyhydroxylated triterpenoid saponins from Eryngium yuccifolium. Phytochemistry 2008, 69, 2070-2080. [CrossRef]

22. Kowalczyk, M.; Masullo, M.; Thiem, B.; Piacente, S.; Stochmal, A.; Oleszek, W. Three new triterpene saponins from roots of Eryngium planum. Nat. Prod. Res. 2014, 28, 653-660. [CrossRef] [PubMed]

23. Derda, M.; Hadaś, E.; Cholewiński, M.; Skrzypczak, Ł.; Grzondziel, A.; Wojtkowiak-Giera, A. Artemisia annua L. as a plant with potential use in the treatment of acanthamoebiasis. Parasitol. Res. 2016, 115, 1635-1639. [CrossRef] [PubMed]

24. Hadaś, E.; Derda, M.; Nawrot, J.; Nowak, G.; Thiem, B. Evaluation of the amoebicidal activities of Centaurea bella, Centaurea daghestanica, Rhaponticum pulchrum and Tanacetum vulgare against pathogenic Acanthamoeba spp. Acta Pol. Pharm. 2017, 74, 1827-1832.

25. Tanveer, T.; Bin-Dukhyil, A.A.; Nawaz, S.; Matin, A. Therapeutic potential of folk plants used against pathogenic and opportunistic free-living amoeba (Acanthamoeba, Balamuthia and Naegleria): A review. Inter. J. Cell Sci. Mol. Biol. 2018, 4, 555628. [CrossRef]

26. Hadaś, R.; Ożarowski, M.; Derda, M.; Thiem, B. The use of extracts from Passiflora spp. in helping the treatment of acanthamoebiasis. Acta Pol. Pharm. 2017, 74, 921-928. [PubMed]

27. Derda, M.; Thiem, B.; Budzianowski, J.; Wojt, W.J.; Wojtkowiak-Giera, A. The evaluation of the amoebicidal activity of Eryngium planum extracts. Acta Pol. Pharm. 2013, 70, 1027-1034.

28. Malatyali, E.; Tepe, B.; Degerli, S.; Berk, S. In vitro amoebicidal activities of Satureja cuneifolia and Melissa officinalis on Acanthamoeba castellanii cysts and trophozoites. Parasitol. Res. 2012, 110, 2175-2180. [CrossRef]

29. Goze, I.; Alim, A.; Dag, S.; Tepe, B. In vitro amoebicidal activity of Salvia staminea and Salvia caespitosa on Acanthamoeba castellani and their cytotoxic potentials on corneal cells. J. Ocul. Pharmacol. Ther. 2009, 25, 293-298. [CrossRef]

30. Malatyali, E.; Tepe, B. In-vitro amoebicidal activity of four Peucedanum species on Acanthamoeba castellanii cysts and trophozoites. Parasitol. Res. 2012, 110, 167-174. [CrossRef]

31. Degerli, S.; Tepe, B.; Celiksoz, A.; Berk, S. In-vitro amoebicidal activity of Origanum syriacum and Origanum laevigatum on Acanthamoeba castellanii cysts and trophozoites. Exp. Parasitol. 2012, 131, 20-24. [CrossRef]

32. Rodríguez-Zaragoza, S.; Ordaz, C.; Avila, G.; Muñoz, J.L. In-vitro evaluation of the amebicidal activity of Buddleia cordata (Loganiaceae, H.B.K.) on several strains of Acanthamoeba. J. Ethnopharmacol. 1999, 66, 327-334. [CrossRef]

33. Dodangeh, S.; Niyyati, M.; Kamalinejad, M. Antiacanthamoeba activities of chloroformic fractions of Trigonella foenum graecum (Seed) and their cytotoxity on mice macrophage cell. Novel. Biomed. 2001, 82, 237-246. 
34. Mahboob, T.; Azlan, A.-M.; Tan, T.-C.; Samudi, C.; Sekaran, S.D.; Nissapatorn, V.; Wiart, C. Anti-encystment and amoebicidal activity of Lonicera japonica Thung. And its major constituent chlorogenic acid in vitro. Asian Pac. J. Trop. Med. 2016, 9, 866-871. [CrossRef] [PubMed]

35. Bittner Fialová, S.; Kello, M.; Čoma, M.; Slobodníková, L.; Drobná, E.; Holková, I.; Garajová, M.; Mrva, M.; Zachar, V.; Lukáč, M. Derivatization of rosmarinic acid enhances its in vitro antitumor, antimicrobial and antiprotozoal properties. Molecules 2019, 24, 1078. [CrossRef]

36. Anwar, A.; Ting, E.L.S.; Anwar, A.; ul Ain, N.; Faizi, S.; Shah, M.R.; Khan, N.A.; Siddiqui, R. Antiamoebic activity of plant-based natural products and their conjugated silver nanoparticles against Acanthamoeba castellanii (ATCC 50492). AMB Express 2020, 10, 24. [CrossRef]

37. Mennai, I.; Hanfer, M.; Esseid, C.; Benayache, S.; Ameddah, S.; Menad, A.; Benayache, F. Chemical composition, in vitro antiparasitic, antimicrobial and antioxidant activities of Frankenia thymifolia Desf. Nat. Prod. Res. 2019, 24, 1-6. [CrossRef]

38. Murashige, T.; Skoog, F. A revised medium for rapid growth and bioassays with tobacco cultures. Physiol. Plant. 1962, 15, 473-497. [CrossRef]

39. Thugawa, H.; Nakabayashi, R.; Mori, T.; Yamada, Y.; Takahashi, M.; Ray, A.; Sugiyama, R.; Yamamoto, H.; Nakaya, T.; Yamazaki, M.; et al. A chemoinformatics approach to characterize metabolomes in stable-isotope-labeled organism. Nat. Meth. 2009, 16, 295-298. [CrossRef]

Sample Availability: Samples of biomass are available from the authors. 